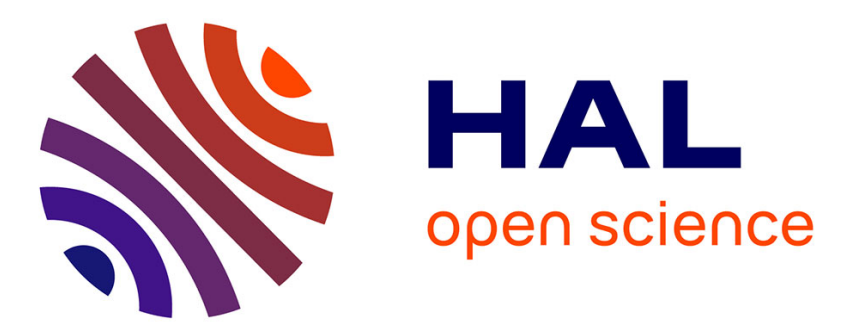

\title{
Non-linear dynamics and contacts of an unbalanced flexible rotor supported on ball bearings
}

Jean-Jacques Sinou

\section{To cite this version:}

Jean-Jacques Sinou. Non-linear dynamics and contacts of an unbalanced flexible rotor supported on ball bearings. Mechanism and Machine Theory, 2009, 44 (9), pp.1713-1732. 10.1016/j.mechmachtheory.2009.02.004 . hal-00411725

\section{HAL Id: hal-00411725 \\ https://hal.science/hal-00411725}

Submitted on 25 Sep 2012

HAL is a multi-disciplinary open access archive for the deposit and dissemination of scientific research documents, whether they are published or not. The documents may come from teaching and research institutions in France or abroad, or from public or private research centers.
L'archive ouverte pluridisciplinaire HAL, est destinée au dépôt et à la diffusion de documents scientifiques de niveau recherche, publiés ou non, émanant des établissements d'enseignement et de recherche français ou étrangers, des laboratoires publics ou privés. 


\title{
Non-linear dynamics and contacts of an unbalanced flexible rotor supported on ball bearings
}

\author{
Jean-Jacques Sinou \\ Laboratoire de Tribologie et Dynamique des Systèmes UMR-CNRS 5513 \\ Ecole Centrale de Lyon, 36 avenue Guy de Collongue \\ 69134 Ecully Cedex, France \\ jean-jacques.sinou@ec-lyon.fr
}

\begin{abstract}
This study deals with the non-linear dynamic response of a flexible rotor supported by ball bearings. The excitation is due to unbalance force. The finite element rotor system is composed of a shaft with one disk, two flexible bearing supports and a ball-bearing element where the non-linearities are due to both the radial clearance and the Herztian contact between races and rolling elements. A numerical analysis is performed to analyze the non-linear behavior of this bearing rotor by using the Harmonic Balance Method with appropriate condensation located only on the non-linear coordinates of the system in order to minimize computer time. The condensation process reduces the original non-linear rotor system by focusing only on the solution of the non-linear equations of the Fourier coefficients associated with the system's non-linear components.

In this study, the procedure is developed for the estimation of the harmonic and super-harmonic responses of the complex rotor system. Consequently, the non-linear unbalance responses and the associated orbits of the bearing rotor will be investigated. Moreover, the transition from contact to no-contact states between rolling elements and races, and the associated restoring contact forces are calculated for different speeds of the unbalanced rotor. Finally, hardening-type nonlinearity or softening-type nonlinearity due to the effects of radial clearance and unbalance mass are examined.
\end{abstract}

\section{Introduction}

One of the most important non-linear mechanical components of rotating machines to be taken into account are bearings due to their considerable influence on the dynamic behavior of rotor systems. For example, various works have studied non-linear behavior due to active magnetic bearings [1-3] and fluid-film bearing [4-8]. The third major type of bearings are the non-linear rolling-bearing elements currently used to support gas turbine engine rotors due to their durability and low power requirements $[4,6,9]$. It is well known that ball bearings are a source of nonlinearities for rotor systems. The nonlinear behavior of rotor systems involving bearing clearances and Hertzian ball-race contact have been studied by several investigators. For example, the dynamic analysis of rotors with bearing clearance was studied by Ehrich $[10,11]$. Sunnersjo [12] studied ball passage vibrations theo- 
retically, taking into account inertia and damping forces. Comparisons with experimentally obtained results were made. Choi and Noah [13] used the harmonic balance method with the discrete Fourier transform procedure to analyze the non-linear behavior of a rotor bearing system. Tiwari et al. [14] studied the non-linear dynamics of a horizontal rigid rotor: the appearance of instability and chaos in the dynamic response are observed both numerically and experimentally. They also studied the effect of radial internal clearance of a ball bearing and the appearance of regions of periodic, subharmonic and chaotic behavior [15]. Later, Harsha et al. [16] proposed to take into account various sources of nonlinearity such as Hertzian contact force, surface waviness and internal radial clearance resulting in the transition from contact to no contact state between races and rolling elements. Periodic, quasiperiodic and chaotic behavior were analyzed in detail. Moreover, Harsha [17] investigated the effect of radial internal clearance for rotor bearing systems in which rolling element bearings show periodic, quasi-periodic and chaotic behavior by considering time response, Poincaré maps and power spectra. Mevel and Guyader [18] observed experimentally the existence of two different routes to chaos in ball bearing dynamic motion. Nataraj and Harsha [19] investigated the nonlinear dynamic behavior of an unbalanced rotor-bearing system due to cage run-out. They indicated that the responses can be placed in three categories: periodic with no sensitivity to initial conditions or small variations of system parameters, quasi-periodic and chaotic with extreme sensitivities to both the initial conditions and small variations in the system parameters. Jang et al. [20] studied the vibration due to ball bearing waviness in a rotating system, taking account of the centrifugal force and gyroscopic moment of the ball.

These studies demonstrated that the nonlinear dynamic response of a balanced rotor supported by rolling element bearings can be very complex and both time consuming and costly to perform when parametric design studies are needed. Therefore, due to the fact that the non linear behavior of rotor systems can be complex, much work has been done on the there has been on the treatment of nonlinear differential equations. More particularly, non-linear methods have been developed to analyse vibration problems $[21,22]$. One of the classical approaches for obtaining the non-linear response of systems is the numerical integration procedure. However, this approach for non-linear models with many degrees of freedom or strong non-linearities can be rather expensive and requires considerable resources both in terms of computation time and data storage. The well-known approximation technique requires an initial assumption about the form of the solution of the non-linear system, i.e. the non-linear solution is approximated by finite Fourier series. In order to study the non-linear dynamics problems of systems with strong non-linearities, the numerical tools most often used are Harmonic Balance Methods and continuation schemes [8,23]. Moreover, if this system can be considered as a linear structure with few additional non-linear elements, it may be of great interest to keep only the non-linear degrees of freedom, by introducing a condensation process to solve only the nonlinear equations associated with the non-linear components of the system [8].

In the present analysis, we propose to demonstrate the efficiency of the Harmonic Balance Method with a condensation process on the non-linear terms in order to study the unbalance responses of a non-linear flexible rotor system with a radial clearance and a Herztian contact between the races and the rolling elements. Secondly, we investigate in particular the evolution of the restoring nonlinear forces on each ball bearing for different rotating speeds. Th effects of the radial clearances and unbalance are investigated and both hardening-type nonlinearity and softening-type nonlinearity are examined.

The paper is divided into three parts. Firstly, the basic concept of the Harmonic Balance Method, the path following continuation based on Lagrangian extrapolation, and the condensation on the non- 
linear degrees-of-freedom are introduced. Secondly, the non-linear rotor system under study is described and developed. The third part presents non-linear analysis of the rotor-bearing system. The evolution of the non-linear responses within the speed range of interest is investigated for different unbalance masses and radial clearances. The associated orbits of the rotor and the stator are calculated. Attention is finally focused on the contact at the bearing-elements: the non-linear contacts for each ball of the bearing are evaluated and discussed for different rotating speeds of the rotor system and for different radial clearances and unbalance masses.

\section{General theory of the harmonic balance method and conden- sation process}

In this section, the harmonic balance method with a condensation process on the non-linear degrees of freedom will be presented.

\subsection{Non-linear method: the Harmonic Balance Method}

For a flexible non-linear rotor, the equations of motion may be written as

$$
\mathbf{M} \ddot{\mathbf{X}}+\mathbf{D} \dot{\mathbf{X}}+\mathbf{K X}=\mathbf{F}_{L}(\mathbf{X}, \dot{\mathbf{X}}, \omega, t)+\mathbf{F}_{N L}(\mathbf{X}, \dot{\mathbf{X}}, \omega, t)=\mathbf{F}(\mathbf{X}, \dot{\mathbf{X}}, \omega, t)
$$

where $\mathbf{F}_{L}$ and $\mathbf{F}_{N L}$ are the linear and non-linear terms of the rotor system. In order to estimate the response of the non-linear system as a truncated Fourier series (if this solution exists), the right-hand side of the system is assumed to be a function that is periodic in time with period $T$. Thus we assume that the non-linear dynamical response of the rotor may be approximated by finite Fourier series with $\omega=\frac{2 \pi}{T}$ the fundamental frequency:

$$
\mathbf{X}(t)=\mathbf{B}_{0}+\sum_{k=1}^{m}\left(\mathbf{B}_{k} \cos (k \omega t)+\mathbf{A}_{k} \sin (k \omega t)\right)
$$

where $m$ is the order of the Fourier series. $\mathbf{B}_{0}, \mathbf{A}_{k}$ and $\mathbf{B}_{k}$ define the unknown coefficients of the finite Fourier series. It can be seen that the Fourier series considered in this study are developed for harmonic and super-harmonic responses of the non-linear rotor system. The number of harmonic coefficients is selected on the basis of the number of significant harmonics expected in the non-linear dynamical response. Generally speaking, harmonic components become less significant when $m$ increases. Moreover, we assume that the vector force $\mathbf{F}(\mathbf{X}, \dot{\mathbf{X}}, \omega, t)$ can be solved in finite Fourier series of order $m$

$$
\mathbf{F}(\mathbf{X}, \dot{\mathbf{X}}, \omega, t)=\mathbf{C}_{0}+\sum_{k=1}^{m}\left(\mathbf{C}_{k} \cos (k \omega t)+\mathbf{S}_{k} \sin (k \omega t)\right)
$$

Substituting equations 2 and 3 in equation 1 yields a set of $(2 m+1) * n$ equations (where $n$ is the number of the degrees-of-freedom for the complete rotor bearing system).

Using the first $n^{\text {th }}$ equations, the constant terms $\mathbf{B}_{0}$ can be determined. We obtain

$$
\mathrm{KB}_{0}=\mathbf{C}_{0}
$$


The $2 m * n$ remaining equations defining the $k^{t h}$ Fourier coefficients $\mathbf{A}_{k}$ and $\mathbf{B}_{k}$ are given by

$$
\left[\begin{array}{cc}
\mathbf{K}-(k \omega)^{2} \mathbf{M} & -k \omega \mathbf{D} \\
k \omega \mathbf{D} & \mathbf{K}-(k \omega)^{2} \mathbf{M}
\end{array}\right]\left[\begin{array}{l}
\mathbf{A}_{k} \\
\mathbf{B}_{k}
\end{array}\right]=\left[\begin{array}{l}
\mathbf{S}_{k} \\
\mathbf{C}_{k}
\end{array}\right]
$$

However, it may be difficult to determine the Fourier coefficients $\mathbf{C}_{0}$, $\mathbf{S}_{k}$ and $\mathbf{C}_{k}$ (with $1 \leq k \leq m$ ) from $\mathbf{B}_{0}, \mathbf{A}_{k}$ and $\mathbf{B}_{k}$ (with $1 \leq k \leq m$ ) directly due to the complexity of the non-linearities. Cameron and Griffin [23] suggested that the truncated Fourier expression of $\mathbf{F}$ should be calculated by applying an Alternate/Frequency Time domain method (AFT-method)

$$
\left[\mathbf{B}_{0} \mathbf{A}_{1} \mathbf{B}_{1} \cdots \mathbf{A}_{m} \mathbf{B}_{m}\right] \stackrel{F F T^{-1}}{\rightarrow} \mathbf{X}(t) \quad \Rightarrow \quad \mathbf{F}(\mathbf{X}, \dot{\mathbf{X}}, \omega, t) \stackrel{F F T}{\rightarrow} \quad\left[\mathbf{C}_{0} \mathbf{S}_{1} \mathbf{C}_{1} \cdots \mathbf{S}_{m} \mathbf{C}_{m}\right]
$$

Following this, the $(2 m+1) * n$ non-linear equations of motion 4 and 5 can be solved by using a non-linear system of equations solver such as the Broyden method [24].

\subsection{The path following continuation}

In the field of rotating machinery, the behaviour of systems is often calculated for different operational speeds of interest while all the other parameters are kept constant. In this case, it may be useful to apply predictor and corrector mechanisms in order to estimate the non-linear response of the rotorbearing system when rotation speed increases or decreases.

We assume that four previous non-linear responses of the rotor-bearing system are obtained. Based on these points, the estimated point on the solution branch can be predicted at a given arc length by applying the Lagrangian polynomial extrapolation method. Any point on the solution branch is represented by $\left(\mathbf{X}_{i}, \omega_{i}\right)$ where $\mathbf{X}_{i}$ and $\omega_{i}$ define the Fourier coefficients and the parameter (i.e. the rotating speed of the rotor-bearing system). Then, the arc length between two consecutive points $\left(\mathbf{X}_{i}, \omega_{i}\right)$ and $\left(\mathbf{X}_{i+1}, \omega_{i+1}\right)$ can be given by

$$
\Delta S_{i+1}=\sqrt{\left(\left(\mathbf{X}_{i+1}-\mathbf{X}_{i}\right)^{T}\left(\mathbf{X}_{i+1}-\mathbf{X}_{i}\right)+\left(\omega_{i+1}-\omega_{i}\right)^{2}\right)} \quad i=0, \ldots, 2
$$

with the arc length parameters given by $\Delta S_{0}=0, S_{1}=\Delta S_{1}, S_{2}=S_{1}+\Delta S_{2}, S_{3}=S_{2}+\Delta S_{3}$ and $S_{4}=S_{3}+\Delta S$.

The estimation of the following point at distance $\Delta S$ can be predicted by using the Lagrangian extrapolation scheme

$$
\left[\begin{array}{c}
\mathbf{X}_{4} \\
\omega_{4}
\end{array}\right]=\sum_{i=1}^{3} \prod_{j=0, i \neq j}^{3}\left(\frac{S_{3}-S_{j}}{S_{i}-S_{j}}\right)\left[\begin{array}{c}
\mathbf{X}_{i} \\
\omega_{i}
\end{array}\right] \quad i=0, \ldots, 3
$$

\subsection{Condensation procedure}

If a non-linear system consists of an $n$-degree of freedom system with non-linear forces associated with $q$ of these degrees of freedom, this system may be considered as a linear structure with $p=n-q$ degrees of freedom and having several additional non-linear elements. Therefore it may be of great interest to keep only the $q$ non-linear degrees of freedom $[8,25]$. Equation 1 can be re-ordered by considering the linear transformation $\mathbf{X}=\mathbf{P Y}=\mathbf{P}\left[\begin{array}{ll}\mathbf{Y}^{p} & \mathbf{Y}^{q}\end{array}\right]^{T}$ where $\mathbf{Y}^{p}$ and $\mathbf{Y}^{q}$ contain the $p$ 
linear degrees of freedom and the $q$ non-linear degrees of freedom, respectively. Therefore the nonlinear equations 1 are transformed by

$$
\left[\begin{array}{ll}
\tilde{\mathbf{M}}_{p p} & \tilde{\mathbf{M}}_{p q} \\
\tilde{\mathbf{M}}_{q p} & \tilde{\mathbf{M}}_{q q}
\end{array}\right]\left[\begin{array}{c}
\ddot{\mathbf{Y}}^{p} \\
\ddot{\mathbf{Y}}^{q}
\end{array}\right]+\left[\begin{array}{cc}
\tilde{\mathbf{D}}_{p p} & \tilde{\mathbf{D}}_{p q} \\
\tilde{\mathbf{D}}_{q p} & \tilde{\mathbf{D}}_{q q}
\end{array}\right]\left[\begin{array}{c}
\dot{\mathbf{Y}}^{p} \\
\dot{\mathbf{Y}}^{q}
\end{array}\right]+\left[\begin{array}{cc}
\tilde{\mathbf{K}}_{p p} & \tilde{\mathbf{K}}_{p q} \\
\tilde{\mathbf{K}}_{q p} & \tilde{\mathbf{K}}_{q q}
\end{array}\right]\left[\begin{array}{c}
\mathbf{Y}^{p} \\
\mathbf{Y}^{q}
\end{array}\right]=\left[\begin{array}{c}
\tilde{\mathbf{F}}^{p} \\
\tilde{\mathbf{F}}^{q}
\end{array}\right]
$$

Here, we present the condensation procedure used to obtain the Fourier coefficients associated with the non-linear and linear elements of the complete system.

\subsubsection{Determination of the constant terms $\mathrm{B}_{0}^{q}$ associated with the non-linear elements}

By substituting equations 2 and 3 in equations 9, and by only equating coefficients for the constant terms corresponding to the first linear equation of the system 4 , we obtain

$$
\left[\begin{array}{ll}
\tilde{\mathbf{K}}_{p p} & \tilde{\mathbf{K}}_{p q} \\
\tilde{\mathbf{K}}_{q p} & \tilde{\mathbf{K}}_{q q}
\end{array}\right]\left[\begin{array}{l}
\mathbf{B}_{0}^{p} \\
\mathbf{B}_{0}^{q}
\end{array}\right]=\left[\begin{array}{c}
\mathbf{C}_{0}^{p} \\
\mathbf{C}_{0}^{q}
\end{array}\right]
$$

where $\mathbf{B}_{0}^{p}$ and $\mathbf{B}_{0}^{q}$ are the vectors with the $p$ linear and $q$ non-linear degrees of freedom of the system, respectively.

By eliminating $\mathbf{B}_{0}^{p}$ from the latter equation, $\mathbf{B}_{0}^{q}$ is given by

$$
\mathbf{B}_{0}^{q}=\left(\tilde{\mathbf{K}}_{q q}-\tilde{\mathbf{K}}_{q p} \tilde{\mathbf{K}}_{p p}^{-1} \tilde{\mathbf{K}}_{p q}\right)^{-1}\left(\mathbf{C}_{0}^{q}-\tilde{\mathbf{K}}_{q p} \tilde{\mathbf{K}}_{p p}^{-1} \mathbf{C}_{0}^{p}\right)
$$

\subsubsection{Determination of the sine Fourier coefficients $\mathrm{A}_{k}^{q}$ associated with the non-linear elements}

Then, by equating coefficients for the cosine terms for the $k^{\text {th }}$ harmonic of equations 5 we obtain

$$
k \omega \tilde{\mathbf{D}} \mathbf{A}_{k}+\left(\tilde{\mathbf{K}}-(k \omega)^{2} \tilde{\mathbf{M}}\right) \mathbf{B}_{k}=\mathbf{C}_{k}
$$

Similarly, by equating coefficients for the sine terms for the $k^{t h}$ harmonic of equations 5 we obtain

$$
\left(\tilde{\mathbf{K}}-(k \omega)^{2} \tilde{\mathbf{M}}\right) \mathbf{A}_{k}-k \omega \tilde{\mathbf{D}} \mathbf{B}_{k}=\mathbf{S}_{k}
$$

Then, the determination of the Fourier coefficients $\mathbf{A}_{k}$ can be obtained by eliminating the Fourier coefficients $\mathbf{B}_{k}$ from equation 12 and 13. By premultiplying equations 12 by $\left(\tilde{\mathbf{K}}-(k \omega)^{2} \tilde{\mathbf{M}}\right)^{-1}$ and substituting in equations 13 , we obtain

$$
\left(\tilde{\mathbf{K}}-(k \omega)^{2} \tilde{\mathbf{M}}+k \omega \tilde{\mathbf{D}}\left(\tilde{\mathbf{K}}-(k \omega)^{2} \tilde{\mathbf{M}}\right)^{-1} k \omega \tilde{\mathbf{D}}\right) \mathbf{A}_{k}=\mathbf{S}_{k}+k \omega \tilde{\mathbf{D}}\left(\tilde{\mathbf{K}}-(k \omega)^{2} \tilde{\mathbf{M}}\right)^{-1} \mathbf{C}_{k}
$$

By introducing

$$
\begin{gathered}
\mathbf{T}_{k}=\tilde{\mathbf{K}}-(k \omega)^{2} \tilde{\mathbf{M}}+k \omega \tilde{\mathbf{D}}\left(\tilde{\mathbf{K}}-(k \omega)^{2} \tilde{\mathbf{M}}\right)^{-1} k \omega \tilde{\mathbf{D}} \\
\mathbf{W}_{k}=\mathbf{S}_{k}+k \omega \tilde{\mathbf{D}}\left(\tilde{\mathbf{K}}-(k \omega)^{2} \tilde{\mathbf{M}}\right)^{-1} \mathbf{C}_{k}
\end{gathered}
$$

equation 14 may be rewritten in partitioned form (as done previously for the constant terms)

$$
\left[\begin{array}{ll}
\mathbf{T}_{k, p p} & \mathbf{T}_{k, p q} \\
\mathbf{T}_{k, q p} & \mathbf{T}_{k, q q}
\end{array}\right]\left[\begin{array}{c}
\mathbf{A}_{k}^{p} \\
\mathbf{A}_{k}^{q}
\end{array}\right]=\left[\begin{array}{c}
\mathbf{W}_{k}^{p} \\
\mathbf{W}_{k}^{q}
\end{array}\right]
$$

Finally, the Fourier coefficients $\mathbf{A}_{k}^{q}$ can be determined by eliminating the Fourier coefficients $\mathbf{A}_{k}^{p}$ from the previous equations. After calculation, we obtain

$$
\mathbf{A}_{k}^{q}=\left(\mathbf{T}_{k, q q}-\mathbf{T}_{k, q p} \mathbf{T}_{k, p p}^{-1} \mathbf{T}_{k, p q}\right)^{-1}\left(\mathbf{W}_{k}^{q}-\mathbf{T}_{k, q p} \mathbf{T}_{k, p p}^{-1} \mathbf{W}_{k}^{p}\right)
$$




\subsubsection{Determination of the cosine Fourier coefficients $\mathrm{B}_{k}^{q}$ associated with the non-linear ele- ments}

The same procedure can now be applied to the determination of the Fourier coefficients $\mathbf{B}_{k}^{q}$. By eliminating the Fourier coefficients $\mathbf{A}_{k}$ from equation 12 and 13 by premultiplying equations 13 by $\left(\tilde{\mathbf{K}}-(k \omega)^{2} \tilde{\mathbf{M}}\right)^{-1}$ and substituting in equations 13 , we obtain

$$
\left(\tilde{\mathbf{K}}-(k \omega)^{2} \tilde{\mathbf{M}}+k \omega \tilde{\mathbf{D}}\left(\tilde{\mathbf{K}}-(k \omega)^{2} \tilde{\mathbf{M}}\right)^{-1} k \omega \tilde{\mathbf{D}}\right) \mathbf{B}_{k}=\mathbf{C}_{k}-k \omega \tilde{\mathbf{D}}\left(\tilde{\mathbf{K}}-(k \omega)^{2} \tilde{\mathbf{M}}\right)^{-1} \mathbf{S}_{k}
$$

Using the relation 15 and introducing

$$
\mathbf{U}_{k}=\mathbf{C}_{k}-k \omega \tilde{\mathbf{D}}\left(\tilde{\mathbf{K}}-(k \omega)^{2} \tilde{\mathbf{M}}\right)^{-1} \mathbf{S}_{k}
$$

equation 19 can be rewritten in partitioned form

$$
\left[\begin{array}{ll}
\mathbf{T}_{k, p p} & \mathbf{T}_{k, p q} \\
\mathbf{T}_{k, q p} & \mathbf{T}_{k, q q}
\end{array}\right]\left[\begin{array}{l}
\mathbf{B}_{k}^{p} \\
\mathbf{B}_{k}^{q}
\end{array}\right]=\left[\begin{array}{c}
\mathbf{U}_{k}^{p} \\
\mathbf{U}_{k}^{q}
\end{array}\right]
$$

Finally, the Fourier coefficients $\mathbf{B}_{k}^{q}$ can be determined by eliminating the Fourier coefficients $\mathbf{B}_{k}^{p}$ from equations 21 . We obtain

$$
\mathbf{B}_{k}^{q}=\left(\mathbf{T}_{k, q q}-\mathbf{T}_{k, q p} \mathbf{T}_{k, p p}^{-1} \mathbf{T}_{k, p q}\right)^{-1}\left(\mathbf{U}_{k}^{q}-\mathbf{T}_{k, q p} \mathbf{T}_{k, p p}^{-1} \mathbf{U}_{k}^{p}\right)
$$

\subsubsection{Determination of the Fourier coefficients $\mathbf{B}_{0}^{p}, \mathbf{A}_{k}^{p}$ and $\mathbf{B}_{k}^{p}$ associated with the linear ele- ments}

Now, the $(2 m+1) * p$ remaining unknown Fourier coefficients $\mathbf{B}_{0}^{p}, \mathbf{A}_{k}^{p}$ and $\mathbf{B}_{k}^{p}$ can be estimated from equations 10, 17 and 21. After calculations, we obtain

$$
\begin{gathered}
\mathbf{B}_{0}^{p}=\tilde{\mathbf{K}}_{p p}^{-1}\left(\mathbf{C}_{0}^{p}-\tilde{\mathbf{K}}_{p q} \mathbf{B}_{0}^{q}\right) \\
\mathbf{A}_{k}^{p}=\mathbf{T}_{k, p p}{ }^{-1}\left(\mathbf{W}_{k}^{p}-\mathbf{T}_{k, p q} \mathbf{A}_{k}^{q}\right) \\
\mathbf{B}_{k}^{p}=\mathbf{T}_{k, p p}{ }^{-1}\left(\mathbf{U}_{k}^{p}-\mathbf{T}_{k, p q} \mathbf{B}_{k}^{q}\right)
\end{gathered}
$$

Moreover, it can be seen that the same condensation process should be applied to the linear elements if the number of linear components is low compared to the number of non-linear components. After calculation, we obtain the following relations

$$
\begin{gathered}
\mathbf{B}_{0}^{p}=\left(\tilde{\mathbf{K}}_{p p}-\tilde{\mathbf{K}}_{p q} \tilde{\mathbf{K}}_{q q}^{-1} \tilde{\mathbf{K}}_{q p}\right)^{-1}\left(\mathbf{C}_{0}^{p}-\tilde{\mathbf{K}}_{p q} \tilde{\mathbf{K}}_{q q}^{-1} \mathbf{C}_{0}^{q}\right) \\
\mathbf{A}_{k}^{p}=\left(\mathbf{T}_{k, p p}-\mathbf{T}_{k, p q} \mathbf{T}_{k, q q}^{-1} \mathbf{T}_{k, q p}\right)^{-1}\left(\mathbf{W}_{k}^{p}-\mathbf{T}_{k, p q} \mathbf{T}_{k, q q}^{-1} \mathbf{W}_{k}^{q}\right) \\
\mathbf{B}_{k}^{p}=\left(\mathbf{T}_{k, p p}-\mathbf{T}_{k, p q} \mathbf{T}_{k, q q}^{-1} \mathbf{T}_{k, q p}\right)^{-1}\left(\mathbf{U}_{k}^{p}-\mathbf{T}_{k, p q} \mathbf{T}_{k, q q}^{-1} \mathbf{U}_{k}^{q}\right)
\end{gathered}
$$

For the latter case, the $(2 m+1) * q$ remaining unknowns Fourier coefficients $\mathbf{B}_{0}^{q}, \mathbf{A}_{k}^{q}$ and $\mathbf{B}_{k}^{q}$ associated with the non-linear components can be deduced from equations 10,17 and 21. 


\subsubsection{Complete process for the determination of the Fourier coefficients and the non-linear dynamical response of the system}

In conclusion, the Fourier coefficients $\mathbf{B}_{0}^{q}, \mathbf{A}_{k}^{q}$ and $\mathbf{B}_{k}^{q}$ associated with the non-linear components of the system are first determined by considering the $(2 m+1) * q$ equations 11,18 and 22 . Secondly, determination of the Fourier $\mathbf{B}_{0}^{p}, \mathbf{A}_{k}^{p}$ and $\mathbf{B}_{k}^{p}$ associated with the linear components of the system are obtained by considering $(2 m+1) * p$ relations 23,24 and 25 . Then, the calculations of the Fourier coefficients $\mathbf{C}_{0}, \mathbf{S}_{k}$ and $\mathbf{C}_{k}$ (with $1 \leq k \leq m$ ) from $\mathbf{B}_{0}=\left[\begin{array}{ll}\mathbf{B}_{0}^{p} & \mathbf{B}_{0}^{q}\end{array}\right]^{T}, \mathbf{A}_{k}=\left[\begin{array}{ll}\mathbf{A}_{k}^{p} & \mathbf{A}_{k}^{q}\end{array}\right]^{T}$ and $\mathbf{B}_{k}=\left[\begin{array}{ll}\mathbf{B}_{k}^{p} & \mathbf{B}_{k}^{q}\end{array}\right]^{T}$ are obtained by using the alternate frequency/time approach (see equations 6 ).

Therefore the previous equations 11, 18 and 22 can be solved by a solver such as the Broyden method [24].

To aid the reader's comprehension, it can be seen that for a general non-linear system, certain linear degrees-of-freedom of the vector $\mathbf{Y}^{p}$ can be transferred and added to the vector $\mathbf{Y}^{q}$ of the non-linear degree-of-freedom without loosing the general process presented previously. This operation can be very interesting if keeping the physical linear degree-of-freedom is necessary for the study (i.e. the non-linear behavior of the chosen physical linear degree-of-freedom can be obtained directly by using relations 18 and 22, without calculating expressions 24 and 25). However, in this case, the size of the vector $\mathbf{Y}^{q}$ has been increased, thereby increasing calculation time and storage requirements.

\section{Application to the flexible rotor-bearing system}

In this section, the harmonic balance method with the condensation procedure on the non-linear coordinates will be applied to the non-linear rotor-bearing system shown in Figure 1.

\subsection{Description of the flexible rotor system}

Firstly, the complete modeling of the rotor-bearing system is presented. The classical equation of motion of the shaft and disc elements will be briefly described. Then, the description and global expressions of the rolling element bearings will be given in detail by considering the kinematics of the rolling elements, the internal clearance and the Hertz contact nonlinearity.

\subsubsection{Shaft elements}

The shaft is modeled by 13 Timoshenko beam elements with circular cross sections. Each Timoshenko beam finite element has four degrees of freedoms at each node:

$$
\left(\mathbf{M}_{T}^{b}+\mathbf{M}_{R}^{b}\right) \ddot{\mathbf{X}}^{b}+\left(\mathbf{C}^{b}+\omega \mathbf{G}^{b}\right) \dot{\mathbf{X}}^{b}+\mathbf{K}^{b} \mathbf{X}^{b}=\mathbf{0}
$$

where $\mathbf{M}_{T}^{b}$ and $\mathbf{M}_{R}^{b}$ are the translational and rotary mass matrices of the shaft element. $\mathbf{C}^{b}, \mathbf{G}^{b}$ and $\mathbf{K}^{\mathbf{b}}$ are the external damping, gyroscopic, and stiffness matrices respectively. $\omega$ is the rotational speed. External damping is taken as classical for the sake of simplicity by considering Rayleigh's expression $\mathbf{C}^{b}=\alpha\left(\mathbf{M}_{T}^{b}+\mathbf{M}_{R}^{b}\right)+\beta \mathbf{K}^{b}$ where $\alpha$ and $\beta$ are constant factors of proportionality and internal rotor damping has been neglected. 


\subsubsection{Rigid disc}

The disk is modeled as a rigid disk and can be written as

$$
\left(\mathbf{M}_{T}^{d}+\mathbf{M}_{R}^{d}\right) \ddot{\mathbf{X}}^{d}+\omega \mathbf{G}^{d} \dot{\mathbf{X}}^{d}=\mathbf{F}^{d}
$$

where $\mathbf{M}_{T}^{d}, \mathbf{M}_{R}^{d}$ and $\mathbf{G}^{d}$ are the translational mass, rotary mass and gyroscopic matrices respectively. $\mathbf{F}^{d}$ defines the unbalance or any other excitation on the disc.

\subsubsection{Flexible supports and coupling}

The flexible supports (see Section A and B in Figure 1) and coupling (see Section C in Figure 1) situated at the two locations of the two rolling-element bearings and the right end of the shaft are modeled as two-node linear elastic spring elements. They are formulated from the following equations of motion:

$$
\mathbf{M}^{s} \ddot{\mathbf{X}}^{s}+\mathbf{K}^{s} \mathbf{X}^{s}=\mathbf{F}^{s}
$$

where $\mathbf{M}^{s}, \mathbf{K}^{s}$ and $\mathbf{F}^{s}$ are the elementary mass matrix, stiffness matrix, and external load vector.

\subsubsection{The rolling bearing}

A schematic diagram of the rolling element bearing with its fixed frame of reference and the spin direction of the rotor is shown in Figure 2. The rolling bearing is modeled as a two degrees of freedom bearing with radial clearance and Hertz contact between races and rolling elements. In this study, the outer race of the ball bearing is assumed to be fixed to the flexible support and the inner race is assumed to be fixed to the shaft.

The precessional angular position of the center of the ball is given by (Harsha et al. [16] and Tiwari et al. [14])

$$
\omega_{\text {cage }}=\omega\left(\frac{R_{i}}{R_{i}+R_{o}}\right)
$$

where $R_{i}$ and $R_{o}$ are the inner and outer race radii. $\omega$ defines the rotational speed of the rotor. The varying compliance frequency is

$$
\omega_{V C}=\omega_{\text {cage }} N_{b}=\omega N_{b}\left(\frac{R_{i}}{R_{i}+R_{o}}\right)
$$

where $N_{b}$ defines the number of rolling elements for the rolling bearing.

By considering that the angular spacing of the rolling elements is constant, the angular space between two balls is equal to $\Delta \theta=\frac{2 \pi}{N_{b}}$ and each ball is located by its angular position changing with time as the shaft rotates

$$
\theta_{k}=\frac{2 \pi}{N_{b}}(k-1)+\omega_{\text {cage }} t \quad, \quad k=1, \ldots, N_{b}
$$

Then the relative radial distance $\Delta_{k}$ between the inner and the outer races at the $k^{\text {th }}$ ball position can be expressed by

$$
\Delta_{k}=\left(x_{o}-x_{i}\right) \cos \left(\theta_{k}\right)+\left(y_{i}-y_{o}\right) \sin \left(\theta_{k}\right) \quad, \quad k=1, \ldots, N_{b}
$$

where $x_{i}, x_{o}, y_{i}$ and $y_{o}$ define the horizontal and vertical displacements of the inner and outer races at the $k^{\text {th }}$ ball. 
Considering the position of each rolling element and the local Hertzian contact, the restoring force can be estimated

$$
F_{k}=\left\{\begin{array}{ccc}
K_{H}\left(\Delta_{k}-\delta\right)^{n} & , & \Delta_{k} \geq \delta \\
0 & , & \Delta_{k}<\delta
\end{array}\right.
$$

where $\delta$ is the radial clearance between races and rolling element. $K_{H}$ defines the effective stiffness which is the combined stiffness of a ball in relation to the inner race and outer race contacts

$$
K_{H}=\frac{1}{\frac{1}{K_{i}^{3 / 2}}+\frac{1}{K_{o}^{3 / 2}}}
$$

The material deformations are elastic and the dimensions of the contact area are small compared to the curvature radii of the contacting bodies. As indicated in equation 36, restoring force $F_{k}$ appears if the $k^{\text {th }}$ ball (at the position $\theta_{k}$ ) is loaded (i.e. $\delta_{k} \geq \delta$ ). If $\delta_{k}<\delta$, the $k^{\text {th }}$ ball is not loaded and no restoring force is generated. It is noted that loading is assumed to be in normal direction at the contacting surfaces and that the deformations at the contact surface are small compared to the dimensions of the contact area.

Finally, the global bearing reaction can be obtained by summing the individual restoring force from each of the rolling-element bearings. Then the total restoring force components $F_{X}$ and $F_{Y}$ in $\mathrm{X}$ and Y directions can be expressed as

$$
\begin{aligned}
& F_{X}=-\sum_{k=1}^{N_{b}} F_{k} \cos \left(\theta_{k}\right) \\
& F_{Y}=-\sum_{k=1}^{N_{b}} F_{k} \sin \left(\theta_{k}\right)
\end{aligned}
$$

\subsubsection{The complete flexible rotor system}

After assembling, the general dynamics equations of the complete rotor system can be written in the following

$$
\mathbf{M} \ddot{\mathbf{X}}+(\mathbf{C}+\omega \mathbf{G}) \dot{\mathbf{X}}+\mathbf{K X}=\mathbf{F}_{L}+\mathbf{F}_{N L}
$$

where $\ddot{\mathrm{X}}, \dot{\mathrm{X}}$ and $\mathbf{X}$ are the acceleration, velocity and displacement vectors. $\mathbf{M}$ is the mass matrix, $\mathrm{C}$ is the external damping matrix associated with the non-rotating parts, $\mathrm{G}$ is the gyroscopic matrix and $\mathbf{K}$ is the stiffness matrix. It should be noted that the internal damping has been neglected in this analysis. However, it may be observed that the non-linear method (i.e. the Harmonic Balance Method) works even if the internal damping of the rotor system is taken into account. $\mathbf{F}_{L}$ contains the weight forces and the unbalance forces. $\mathbf{F}_{N L}$ corresponds to the complete non-linear forces due to the rolling-element bearings. $\omega$ defines the rotating frequency of the system.

All the parameters of the rolling-bearing element and the rotor system are given in Tables 1 and 2. 

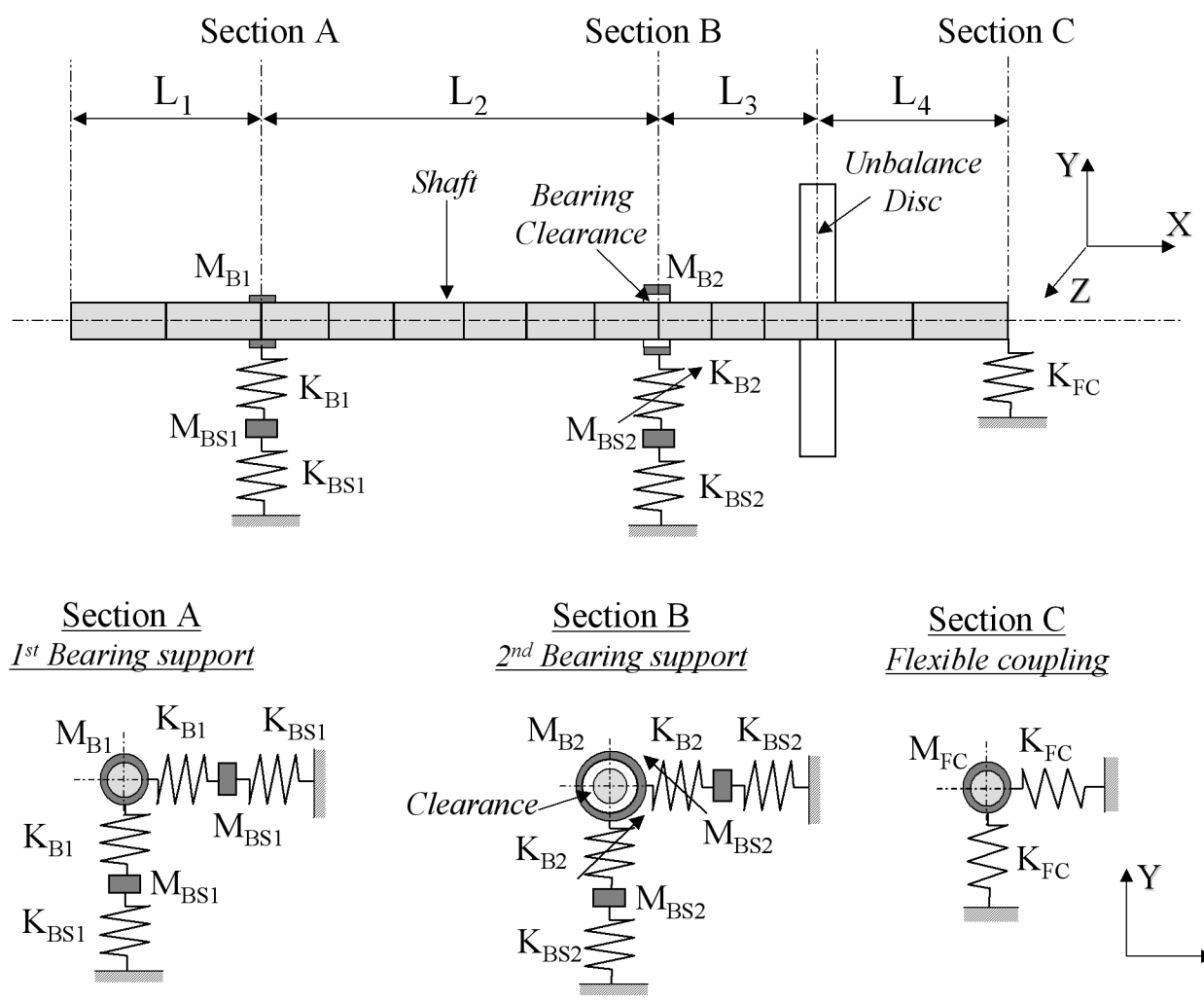

\section{Section C Flexible coupling}

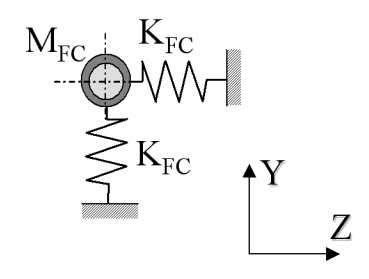

Figure 1: Rotor system

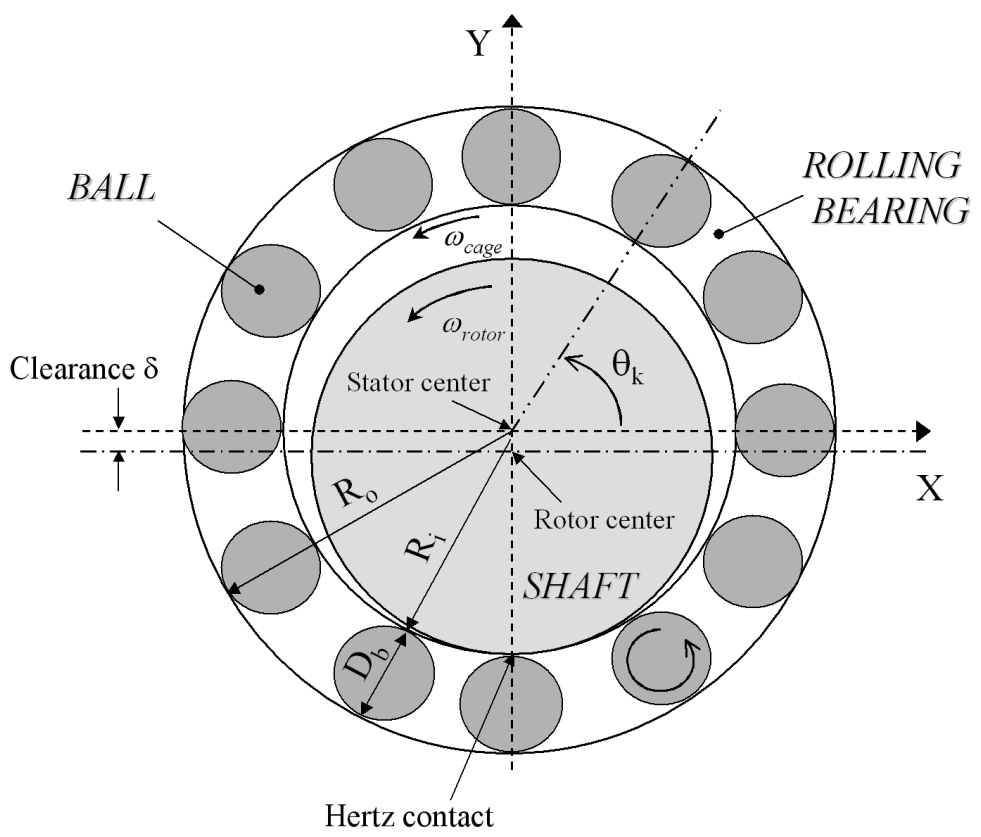

Figure 2: Rolling element bearing and definition of the stator and rotor centers 


\begin{tabular}{ccc}
\hline Notation & Description & Value \\
\hline$R_{d}$ & Ball radius & $5.953 \mathrm{~mm}$ \\
$R_{o}$ & outer race radius & $38.933 \mathrm{~mm}$ \\
$N_{b}$ & number of balls & 14 \\
$\delta$ & radial clearance & $20 \mu \mathrm{m}$ \\
$M_{B 2}$ & rolling element mass & $1.5 \mathrm{~kg}$ \\
$K_{H}$ & load-deflection factor for contact point & $4.6 \times 10^{7} \mathrm{~N} / \mathrm{m}^{3 / 2}$ \\
\hline
\end{tabular}

Table 1: Geometric properties of the rolling-bearing element

\begin{tabular}{ccc}
\hline Notation & Description & Value \\
\hline$D_{\text {shaft }}$ & diameter of the shaft & $0.04 \mathrm{~m}$ \\
$L_{\text {shaft }}$ & length of the shaft & $1.7 \mathrm{~m}$ \\
$L_{1}$ & $1^{\text {st }}$ length section of the shaft & $0.222 \mathrm{~m}$ \\
$L_{2}$ & $2^{\text {nd }}$ length section of the shaft & $1.136 \mathrm{~m}$ \\
$L_{3}$ & $3^{\text {rd }}$ length section of the shaft & $0.146 \mathrm{~m}$ \\
$\rho$ & density & $7800 \mathrm{~kg} / \mathrm{m}^{3}$ \\
$E$ & Young's modulus of elasticity & $2.1 \times 10^{11} \mathrm{~N} / \mathrm{m}^{2}$ \\
$\alpha$ & first Rayleigh damping coefficient & 1.36 \\
$\beta$ & second Rayleigh damping coefficient & $1.75 \times 10^{-5}$ \\
$D_{d i s c / o u t e r}$ & outer diameter of disc & $0.4 \mathrm{~m}$ \\
$D_{\text {disc } / \text { inner }}$ & inner diameter of disc & $0.04 \mathrm{~m}$ \\
$h_{\text {disc }}$ & thickness of disc & $0.02 \mathrm{~m}$ \\
$d_{u}$ & eccentricity of the mass unbalance & $0.2 \mathrm{~m}$ \\
$M_{B 1}$ & $1^{\text {st }}$ rolling element mass & $1.5 \mathrm{~kg}$ \\
$K_{B 1}$ & $1^{s t}$ rolling element stiffness & $7 \times 10^{8} \mathrm{~N} / \mathrm{m}$ \\
$M_{B 2}$ & $2^{\text {nd }}$ rolling element mass & $1.5 \mathrm{~kg}$ \\
$K_{B 2}$ & load-deflection factor for point contact & $4.6 \times 10^{7} \mathrm{~N} / \mathrm{m}^{3 / 2}$ \\
$M_{B S 1}$ & $1^{\text {st }}$ bearing support mass & $6 \mathrm{~kg}$ \\
$K_{B S 1}$ & $1^{\text {st }}$ bearing support stiffness & $3.8 \times 10^{6} \mathrm{~N} / \mathrm{m}$ \\
$M_{B S 2}$ & $2^{\text {nd }}$ bearing support mass & $6 \mathrm{~kg}$ \\
$K_{B S 2}$ & $2^{\text {nd }}$ bearing support stiffness & $3.8 \times 10^{6} \mathrm{~N} / \mathrm{m}$ \\
$M_{F C}$ & flexible coupling mass & $0.73 \mathrm{~kg}$ \\
$K_{F C}$ & flexible coupling stiffness & $5.75 \times 10^{4} \mathrm{~N} / \mathrm{m}$ \\
\hline & & \\
\hline
\end{tabular}

Table 2: Parametric values of the flexible rotor system 


\subsection{Numerical studies}

As explained previously, the rolling-bearing system investigated here is composed of a horizontal flexible shaft of $1700 \mathrm{~mm}$ length with a circular cross section of $40 \mathrm{~mm}$. The shaft has one disk with a diameter of $400 \mathrm{~mm}$ and a thickness of $20 \mathrm{~mm}$ attached $196 \mathrm{~mm}$ from the right-end of the shaft. The flexible coupling is added at the right-end of the shaft to connect the motor. The rotor shaft is supported by two bearing supports; the two supports are placed $342 \mathrm{~mm}$ and $1478 \mathrm{~mm}$ respectively from the end of the shaft with the flexible coupling. The second bearing support illustrated in Figure 1 is composed of a rolling bearing with radial clearance and Hertz contact between the races and rolling elements, as previously explained in Section 3.1.4. All the parametric values for the rolling bearing element and the complete rotor are given in Tables 1 and 2.

Here, the non-linear unbalance responses due to the non-linear and linear components will be investigated, after which the non-linear restoring forces and the associated contact evolution at the rolling bearing element will be calculated.

\subsubsection{Non-linear unbalance responses}

The non-linear unbalance responses and contacts of the non-linear components (i.e. the rotor and stator coordinates at the non-linear rolling bearing elements) are obtained by solving the non-linear equations 11, 18 and 22, the non-linear unbalance responses of the linear components being obtained by using the relations 23,24 and 25 .

The number of harmonics chosen for the approximated solution is selected on the basis of the number of significant harmonics expected in the non-linear dynamical response. In this study, the number of significant harmonic components varies with the rotating speed of the rotor due to the relative contribution of the unbalance forces, gravity and restoring contact forces on each ball bearing. However, the number of harmonic components retained in the solution for the following numerical analysis is equal to twelve due to the fact that the harmonic components become less significant for upper orders. The complete vertical and horizontal unbalance responses at the non-linear rotor-bearing element and the contribution of the first twelve orders for the non-linear components are shown in Figures 3 (for a radial clearance of $\delta=10 \mu \mathrm{m}$ and an unbalance mass of $m_{u}=2 g$ ). The non-linear unbalance responses of the linear components of the rotor system at the left end of the shaft are given in Figure 4. These non-linear amplitudes are obtained by considering the previous relations 23, 24 and 25. Firstly, it is shown that the complete non-linear dynamic responses (noted "CP" in Figures) at the non-linear and linear rotor-bearing elements are very complex, with significant contributions not only of the first and second orders (i.e. the blue and red lines) but also the sixth orders (i.e. the grey lines) when the rotating speed is in the range [0-500] rpm. However, it can be seen that in some cases the first and second order approximations of the truncated Fourier series should be enough to obtain a good approximation of the non-linear dynamics of the rolling-bearing rotor system (see, for example, the speed range $\omega=[1000 ; 4000] \mathrm{rpm}$ where the amplitudes of the rotor are maximal).

Moreover, it is clearly shown that the contribution of the $n^{\text {th }}$ upper orders can be significant at low amplitudes and when the rotor is passing through the $\frac{1}{n}$ critical speeds. To facilitate understanding, the forward and backward critical speeds for the first and second modes are situated around 1740rpm and $2050 \mathrm{rpm}$, and $2900 \mathrm{rpm}$ and $3520 \mathrm{rpm}$, respectively. For example, the $7 \times, 6 \times, 5 \times, 4 \times, 3 \times$ and $2 \times$ harmonic components are predominant at 260rpm, 300rpm, 360rpm, 450rpm, 610rpm and $880 \mathrm{rpm}$ (290rpm, 340rpm, 400rpm, 500rpm, 670rpm and 1020rpm, respectively) due to the fact that the rotation speed passes through the subharmonic components of the first backward critical speed (and 
the first forward critical speed, respectively). The same phenomenon can be observed for the second backward and forward critical speeds: when the rotor passes through the $7 \times, 6 \times, 5 \times, 4 \times, 3 \times$ and $2 \times$ harmonic components of the backward or forward critical speeds of the second mode (at 430rpm, 500rpm, 600rpm, 750rpm, 970rpm and 1480rpm for the backward mode, respectively; at 470rpm, 540rpm, 660rpm, 820rpm, 1100rpm and 1720rpm for the forward mode, respectively), the amplitudes of the $n^{\text {th }}$ associated upper orders increase.

The non-linear responses of the rotor at the non-linear rotor-bearing element and at the left end of the shaft are illustrated in Figures 5 for a radial clearance of $\delta=10 \mu \mathrm{m}$ and an unbalance mass of $m_{u}=4 \mathrm{~g}$. It can be seen that the non-linear responses of the rotor system are complex with jump phenomenon for rotor speeds between $\omega=2100 \mathrm{rpm}$ and $\omega=2300 \mathrm{rpm}$. This jump is not only observed for the first order, but also for the second, third, fourth and fifth orders, as illustrated in Figures 5. This phenomenon indicates the "hardening effect" at the first critical speed due to the contribution of the non-linear terms of the rolling bearing. Therefore, for an excitation level about twice as high as that of the initial case, the peak of the first forward critical speed appears for a rotating speed higher than about $7 \%$ with respect to the initial case. The hardening-type nonlinearity on the maximum amplitude of the harmonic components can also be observed. It is then possible to see that two stable solutions coexist when the rotation speed is around [2140:2210] rpm.

As previously seen for the unbalance case $m_{u}=2 g$ (Figures 3 and 4), the global non-linear responses are very complex with the significant presence of the first, second and sixth orders (see the horizontal amplitudes at the left end of the shaft). Even if the non-linear behavior between the two cases ( $m_{u}=2 g$ and $m_{u}=4 g$ ) are different (with the appearance of jump phenomenon for example), the global contributions of each order for the two cases are very similar: the maximum amplitude is observed when the rotation speed exceeds the first critical speed $(\omega=2000 \mathrm{rpm}$ ), the most important contributions are given by the first and second orders when the speed reaches about [1000-4000]rpm, the sixth order makes a significant contribution at low speed (around [0-500]rpm), and the $n^{\text {th }}$ orders appear when the rotor speed reaches around $\frac{1}{n}$ of critical speeds. Finally, it can be seen that the nonlinear responses at the left end of the shaft (see the line " $\mathrm{CP}$ "' in Figures 4 and 5) are governed by the amplitudes of the sixth order when the rotation speed is between [0-1300]rpm: the contribution of the sixth order is most significant when the rotation speed is between [0-500]rpm and one of the highest amplitudes if the rotor speed reaches around [500-1300]rpm.

The orbits at the disc position, left end and middle of the shaft are shown in Figures 6 for various rotation speeds between [300-1500]rpm when the components of the $n^{\text {th }}$ orders have significant contributions. Thus it is clear that the non-linear responses can be very complex with "multiple inside or outside loops"”.

Figures 7 show the complete non-linear amplitudes for the rotor shaft and the two stators of the rolling bearing elements at specific rotation speeds. For each case, the orbits associated with the non-linear degrees of freedom (and thus calculated by using the condensation process and the relation equations 11, 18 and 22) are indicated by red lines. The orbits corresponding to the linear degrees of freedom obtained by using the expressions 23,24 and 25 are indicated by the black lines. The blue line illustrates the deflection of the rotor shaft. To facilitate comprehension, the top subfigures illustrate the rotor amplitudes of the linear and non-linear degrees of freedom, and the stator amplitudes at the rolling bearing element are presented by the lower subfigures (for the linear and non-linear degrees of freedom). It should be noted that the orbits and the deflection of the shaft are presented without the static deflection of the rotor in order to clearly show the complexity of the non-linear response that generally occurs at low amplitudes. 
(a)

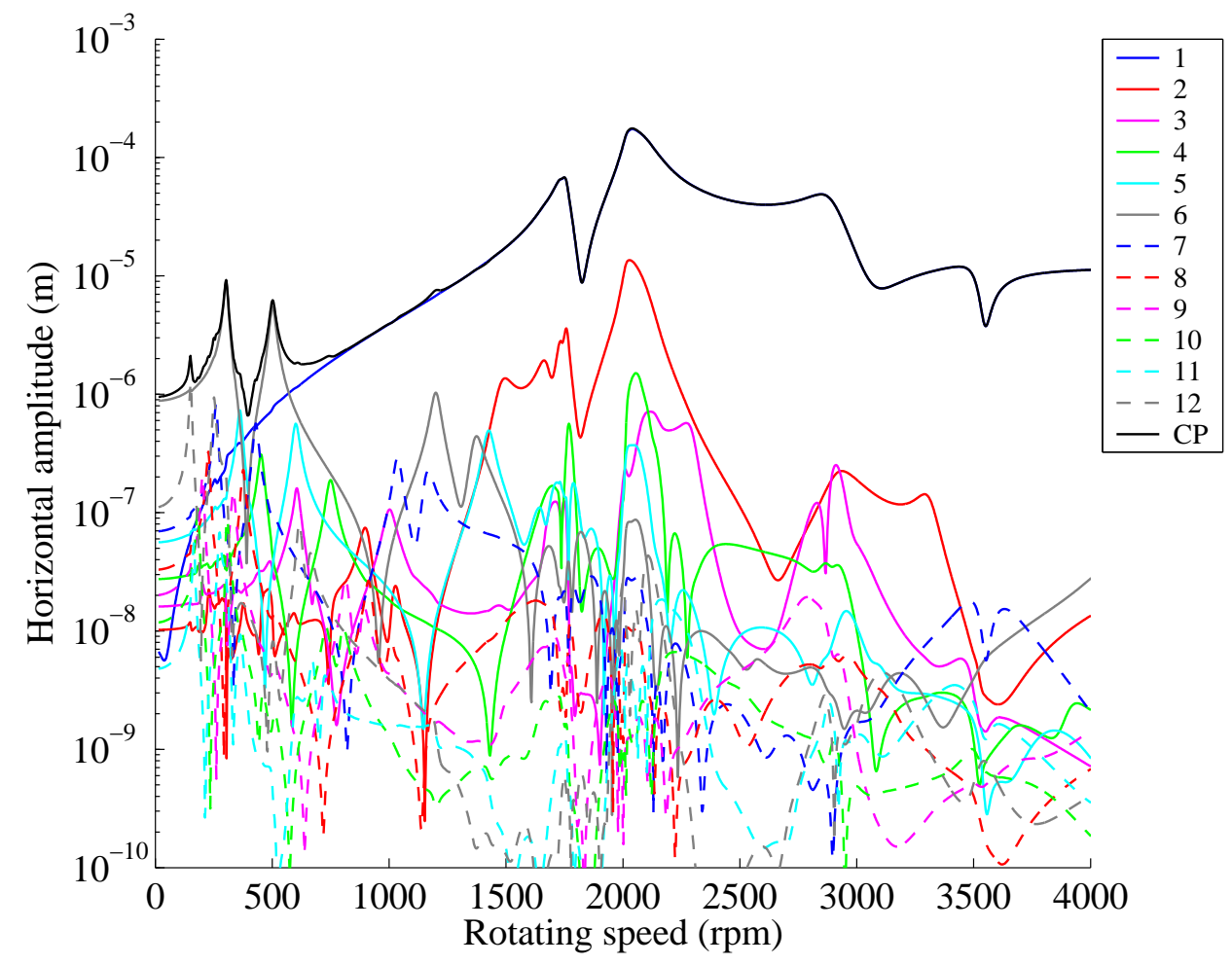

(b)

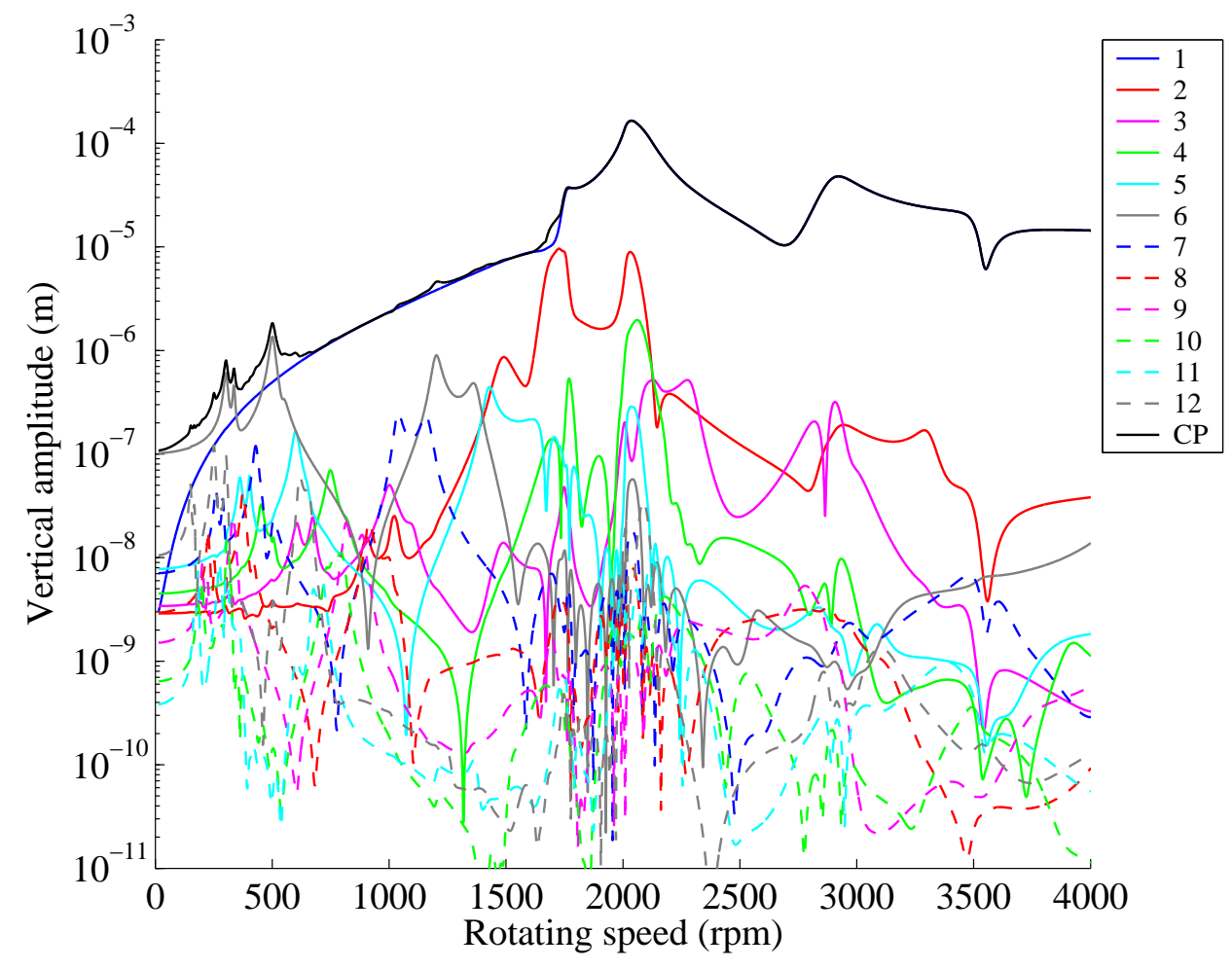

Figure 3: Non-linear unbalance response at the non-linear rotor-bearing element for $\delta=10 \mu \mathrm{m}$ and $m_{u}=2 g$ (a) horizontal (b) vertical 


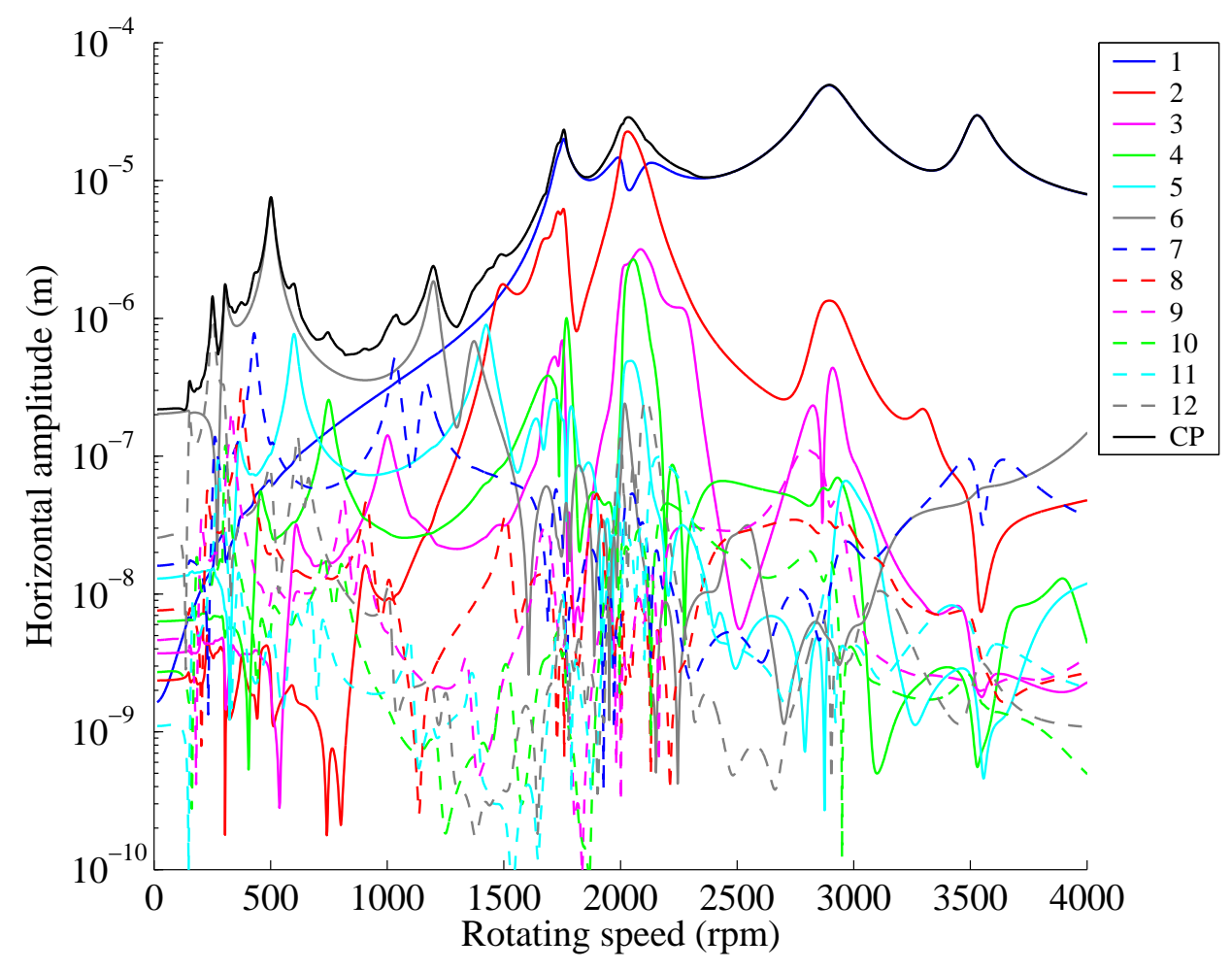

Figure 4: Non-linear horizontal unbalance responses at the linear rotor elements (left end of the shaft) for $\delta=10 \mu m$ and $m_{u}=2 g$

(a)

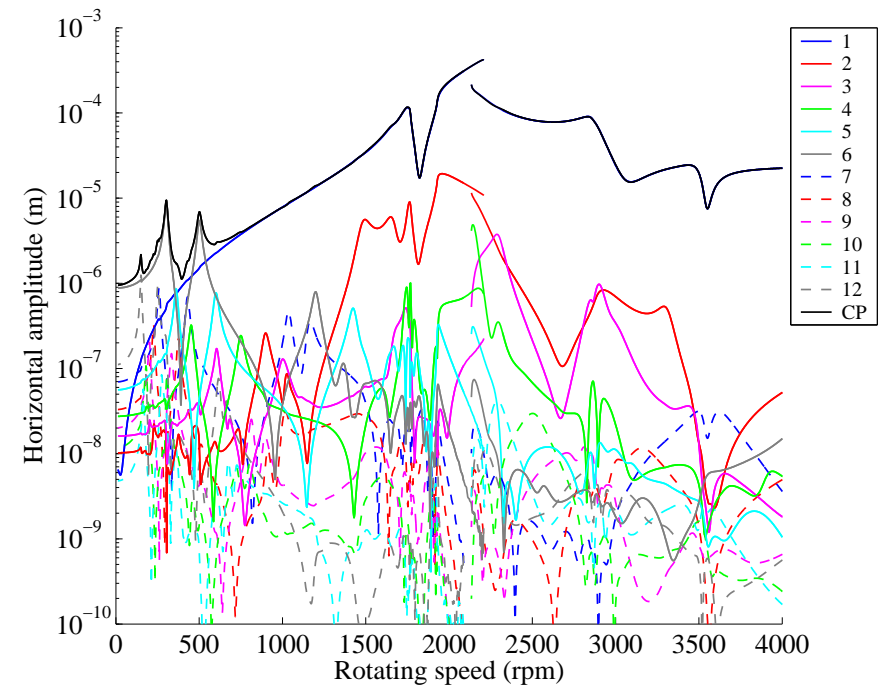

(b)

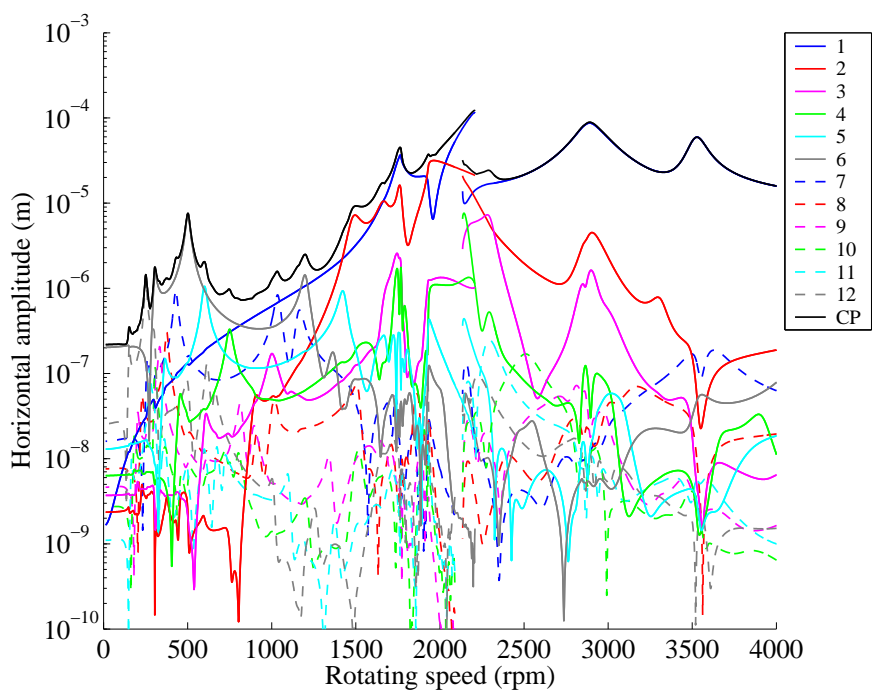

Figure 5: Non-linear horizontal unbalance responses of the rotor for $\delta=10 \mu m$ and $m_{u}=4 g$ (a) at the non-linear rotor-bearing element (b) at the left end of the shaft 
Figures 7 (c) and (d) show the shaft's deflection at the backward and forward first critical speeds (for $\omega=1737 \mathrm{rpm}$ and $\omega=2052 \mathrm{rpm})$. The shaft's deflections for the backward and forward second critical speeds (for $\omega=2932 \mathrm{rpm}$ and $\omega=3526 \mathrm{rpm}$ ) are given in Figures 7 (e) and (f), respectively. It may be observed that the rotor's orbits are very simple and similar to a simple loop when the rotation speed is passing through the second critical speeds. For the first critical speeds, simple loops are shown at the right-end of the shaft and at the disc position. However, inner loops appear for the orbits at the left position, at the middle of the shaft and at the first bearing support. These results are in perfect agreement with the previous evolutions of the Fourier components: the second order of the Fourier series is sufficient to describe the non-linear behavior and the associated orbits of the nonlinear rotor system at the first critical speeds, and the first order gives an adequate approximation of the rotor orbits at the second critical backward and forward critical speeds. Finally, Figures 7 (a) and (b) illustrate that the orbits can be more complex (as previously seen in Figures 6): the contributions of the $n^{\text {th }}$ order are shown for all the shaft orbits. All these orbits shown in Figures 6 and 7 clearly indicate the complexity and variability of the non-linear responses of the rotor-bearing system.

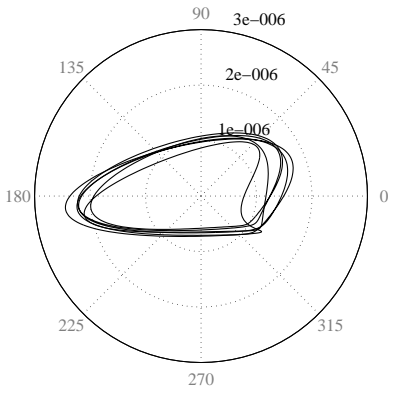

(a) $\omega=300 \mathrm{rpm}$

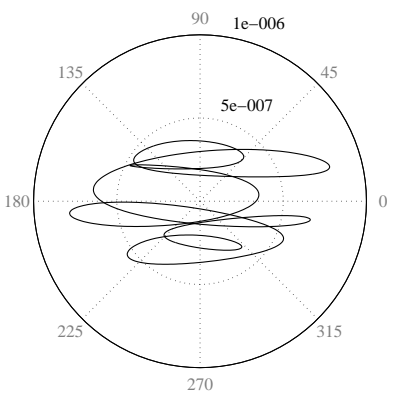

(e) $\omega=740 \mathrm{rpm}$

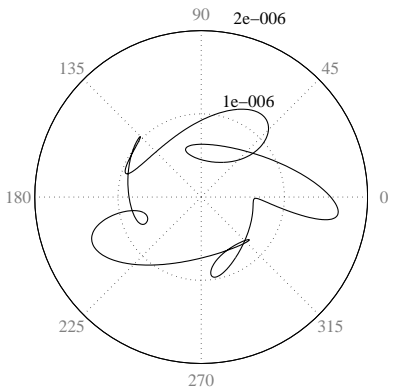

(b) $\omega=460 \mathrm{rpm}$

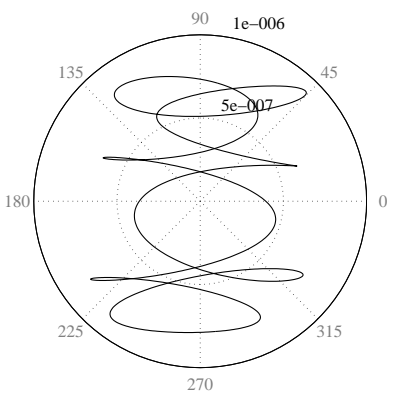

(f) $\omega=900 \mathrm{rpm}$

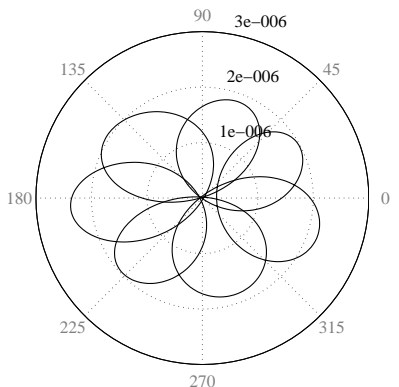

(c) $\omega=500 \mathrm{rpm}$

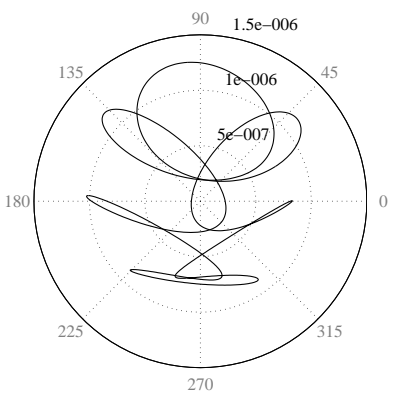

(g) $\omega=1100$ rpm

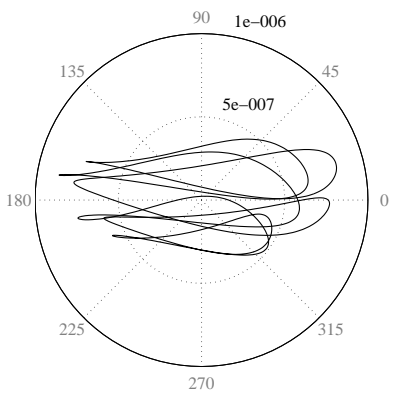

(d) $\omega=660 \mathrm{rpm}$

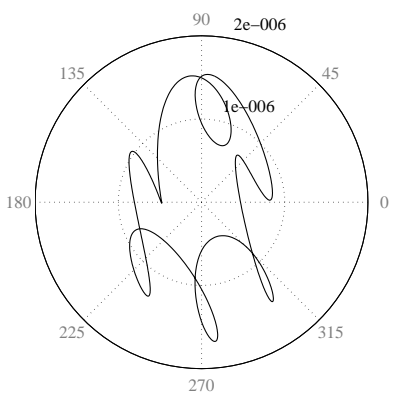

(h) $\omega=1300$ rpm

Figure 6: Evolution of complex orbits for $\delta=10 \mu m$ and $m_{u}=2 g$ (b,c) disc position (d,e,g,h) left end $(\mathrm{a}, \mathrm{f})$ middle of the shaft

\subsubsection{Non-linear contact forces and orbits at the rolling-bearing element}

In this part of the paper, the contacts between races and balls and the associated restoring forces in fixed coordinates (OXY in Figure 1) are investigated in order to understand the relative contribution of the unbalance and gravitational forces for the rotor, the shaft whirling motion, and the associated nonlinear behavior at the rolling-bearing element. Due to the condensation process previously presented and by using the relations 11,18 and 22 , the non-linear behavior of the rotor system is only estimated 

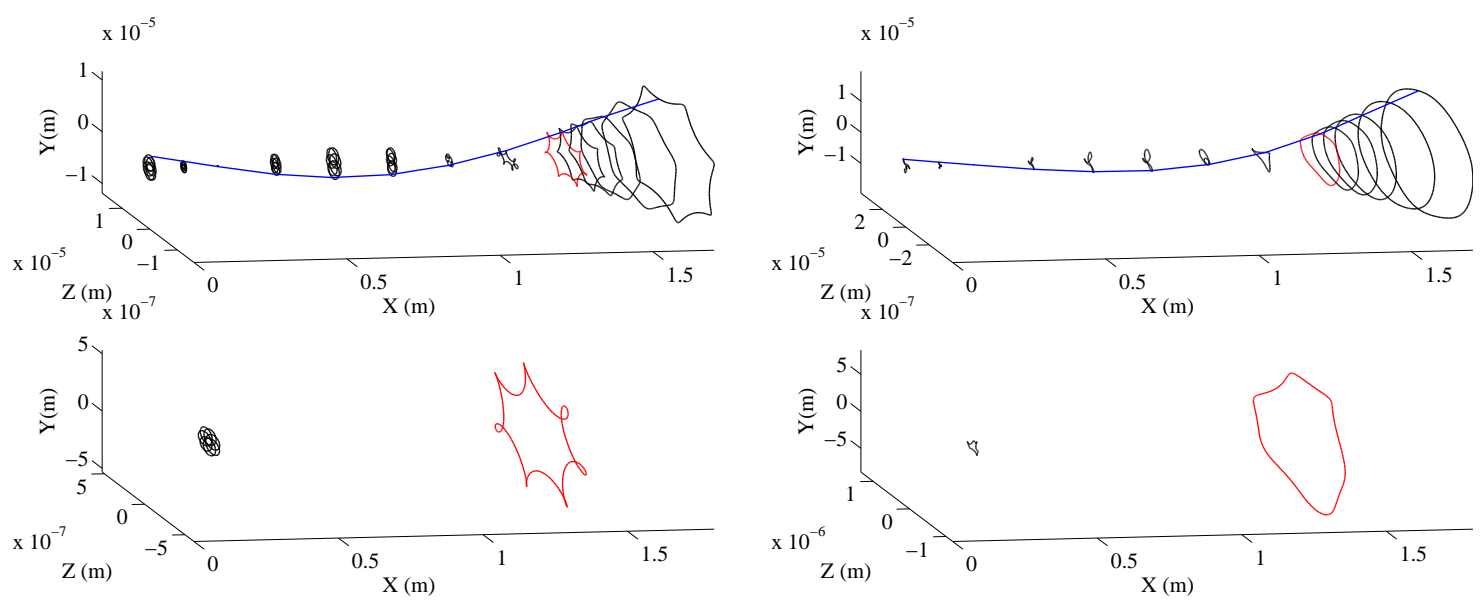

(a) $\omega=1203 \mathrm{rpm}$

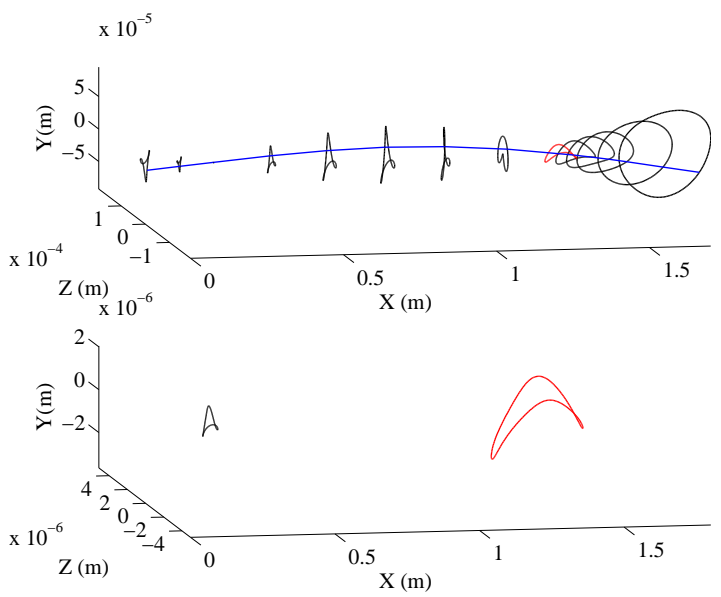

(b) $\omega=1488 \mathrm{rpm}$

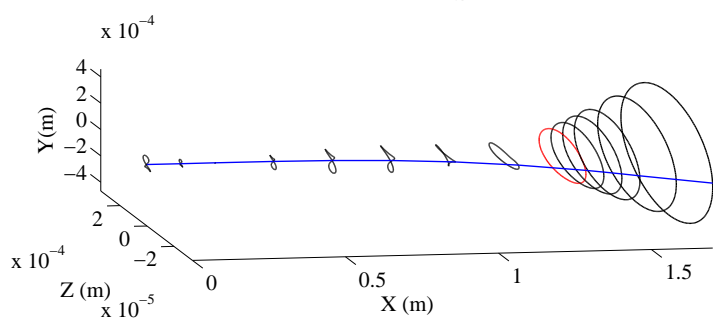

(c) $\omega=1737 \mathrm{rpm}$

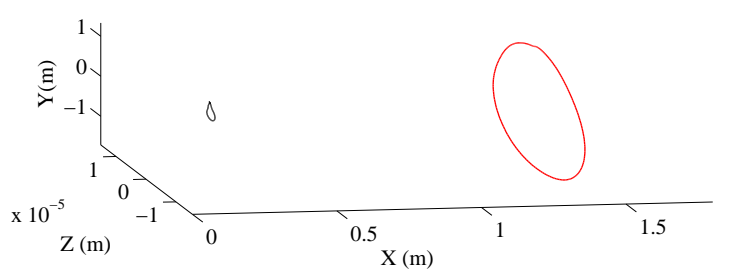

(d) $\omega=2052 \mathrm{rpm}$
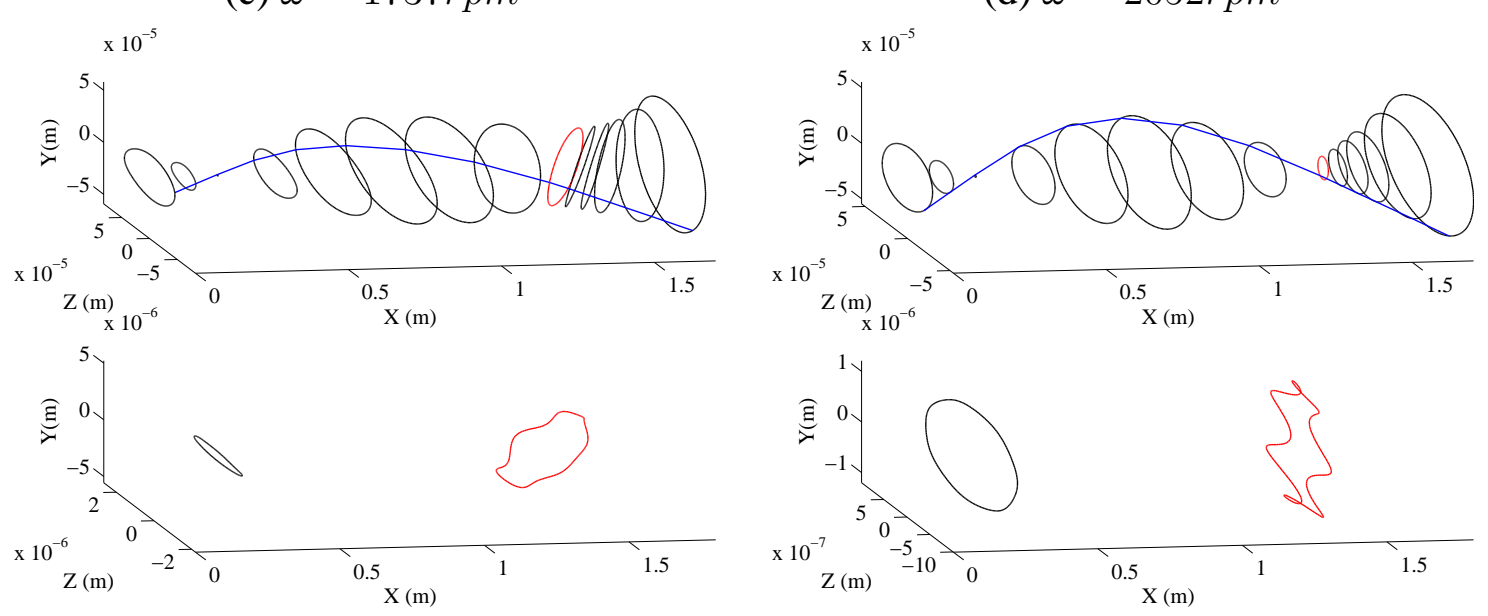

(e) $\omega=2932 \mathrm{rpm}$

(f) $\omega=3526 \mathrm{rpm}$

Figure 7: Evolution of the orbits for $\delta=10 \mu m$ and $m_{u}=2 g$ 
at the non-linear degrees of freedom in this part of the study.

In order to carry out this analysis, the evolution of the contact on each ball is first evaluated and discretized over one period of revolution of the rolling-bearing cage $\omega_{\text {cage }}=\omega\left(R_{i} /\left(R_{i}+R_{o}\right)\right)$ where $R_{i}$ and $R_{o}$ are the outer and inner race radii of the rolling bearing element and $\omega$ defines the rotation speed of the rotor. Secondly, the global restoring force is calculated in the fixed coordinates not only over one period of revolution of the rolling-bearing cage but also over fifty periods, in order to show the envelops of the non-linear contact forces of the rolling bearing element.

Figures 8 and 9 show the evolution of the contact restoring forces for each ball of the bearing for a radial clearance of $\delta=10 \mu \mathrm{m}$, and an unbalance mass of $m_{u}=2 g$ and $m_{u}=4 g$, respectively. The white lines define the limits between the contact and no-contact zones for each ball.

Figures 10 and 11 give the global restoring forces for both cases in the fixed coordinates. The blue lines represent the evolution of the non-linear contact over one period of revolution of the rollingbearing cage. The green surface corresponds to the envelop of the non-linear contact over fifty periods of revolution. The red line indicates that the rotor and stator are in contact with a non-linear force greater than zero. Figures 9 and 11 give contact evolutions for all the speed ranges of interest (i.e. $\omega=[0-4000] \mathrm{rpm}$ and for $m_{u}=4 \mathrm{~g}$ ) whereas Figures 8 and 10 focus on the first critical speeds for $m_{u}=2 g$.

For the first unbalance mass $m_{u}=2 g$, the rotor moves at the bottom of the bearing for different rotation speeds $(\omega=1895 \mathrm{rpm}, \omega=1990 \mathrm{rpm}, \omega=2215 \mathrm{rpm}$ and $\omega=2244 \mathrm{rpm}$ ), as indicated in Figures $10(a, b, e, f)$ with the red lines that define the non-linear contact of the rolling-bearing element in fixed coordinates. Regarding the associated non-linear contacts on each ball of the bearing (see Figures $8(a, b, e, f)$ ), five or six balls are in contact with the outer ring each time and the number of times of contact and non-contact per ball is similar for each ball. For $\omega=1895 \mathrm{rpm}$ and $\omega=2244 \mathrm{rpm}$, the evolution of contact for each ball is due to the revolution of the rolling-bearing cage: the area within which the rolling elements are still in contact with the raceway, generally referred to as the loaded zone, is situated at the bottom of the ball bearings (in fixed coordinates). The value of the non-linear contact force is still high, as shown in Figure 10(a) and (f) (see the green surfaces that indicate the contact over fifty periods of revolution of the rolling-bearing cage). When the rotor exceeds the first forward critical speed (i.e. $\omega=1990-2130 \mathrm{rpm}$ ), the contact value at the bottom of the bearing can be equal to zero (see the green surface in Figures $10(\mathrm{~b}, \mathrm{c}, \mathrm{d}, \mathrm{e})$ ) and the red lines correspond to the full circle. Moreover, the evolutions of the non-linear contacts on each ball become more complex, as indicated in Figures 8(b,c,d,e). The contact and non-contact times per ball can differ considerably from one ball to another and the associated restoring contact forces can increase or decrease during one period of revolution of the rolling-bearing cage. Finally, Figures 10(c) and 8(c) illustrate the case of a complete whirling contact on the rolling element (i.e. the radial clearance in the fixed coordinates is consumed while the red line defines the full "circle"" in Figure 10(c)). It is noted that the value of the non-linear restoring forces is still higher at the bottom of the rolling element. Due to cage rotation, the maximum value of the non-linear contact changes from one ball to another, as indicated in Figure 8(c). Finally, it can be seen that the period of contact/no-contact between the rotor and each ball in this case (i.e. $\omega=2030 \mathrm{rpm}$ and $m_{u}=2 g$, Figure 8(c)) decreases in comparison to the previous cases (i.e. $\omega=1895 \mathrm{rpm}$ or $\omega=2244 \mathrm{rpm}$ for $m_{u}=2 g$, Figures $8(\mathrm{a}, \mathrm{f})$ ).

Then, for the second unbalance mass $m_{u}=4 g$, the same non-linear evolutions are observed around the first critical speed: each ball participates with almost the same contact time over the period of rotation of the rolling-bearing cage and the non-linear contact forces can increase or decrease during one period. When the rotor whirls on the bottom of the bearing, the evolution of the contact forces 
corresponds to one period of revolution of the rolling-bearing cage. At the first critical speed (i.e. $\omega=2100-2200 \mathrm{rpm}$ ), the rotor whirls completely in the rolling-bearing element and the clearance is consumed in the fixed coordinates during its rotation (i.e. the red lines define the "full circle", as shown in Figure 11). Moreover, due to the increase of the unbalance force, the contact on all the rotor-bearing elements (i.e. the "red circle"") is present for a greater speed range and the values of the non-linear contact forces at the top and bottom of the rolling element are more higher (see Figure 11 for $\omega=2195 \mathrm{rpm}$ ). In this case, the unbalance effect is predominant for the non-linear behavior of the contact/no-contact evolutions. As seen in Figure 9 for $\omega=2204 \mathrm{rpm}$ three or four contacts occur per period of revolution of the rolling-bearing cage for each ball. Each ball participates with almost the same contact time over the period of rotation. As previously shown, the period of contact/no-contact between the rotor and each ball is similar to the period of the rotation of the rotor $\omega$.

However, if the rotation speed is around the first critical speed, the contact time and the restoring contact force are very different and complex per period of revolution for each ball (see Figures 9 for $\omega=1919 \mathrm{rpm} \omega=2253 \mathrm{rpm}$ ) even if the rotor and stator orbits are simple and close to a periodic harmonic form (see results of the previous section).

Finally, the non-linear contact forces are given for the speed range of interest $\omega=0-4000 \mathrm{lpm}$ in Figures 11. It can be seen that the non-linear contact over one period of revolution of the cage (i.e. the blue line) or the envelopes of the non-linear contact (i.e. the green surface) are totally different for the different rotation speeds and can be complex in some cases. For example, the non-linear contact can be dissymmetrical on the rotor-bearing element (for example, at the following rotation speeds: $\omega=[1696 ; 1787 ; 2703 ; 2890] \mathrm{rpm}$ ). These dissymmetric non-linear restoring forces at the rolling element are observable at the first and second backward critical speeds where the rotor orbits are elliptical. Then, at low amplitudes, the value of the non-linear force can remain constant due to the fact that the rotor moves on the bottom of the rolling element: see for example Figure 11 for the following rotation speeds $\omega=[296 ; 1595 ; 4000] \mathrm{rpm}$ where the blue lines (i.e. evolution of the non-linear contact forces over one period of revolution of the rolling-bearing cage) are similar to the green surface (i.e. evolution of the non-linear contact forces over fifty periods of revolution of the rolling-bearing cage). As explained previously, the contact and non-contact times per ball and the value of the non-linear forces are similar from one ball to another, in this case due to the rotation of the rolling cage.

Finally, it can be seen that the non-linear contact force at the rolling element can be different for a given rotation speed if in run-up or run-down configurations due to the jump phenomenon. This fact is illustrated in Figure 12 for $m_{u}=4 g$ and $\delta=10 \mu m$.

\subsubsection{Influence of the mass unbalance and the radial clearance}

In this section, the influence of the mass unbalance $m_{u}$ and the radial clearance $\delta$ on the non-linear responses, the Fourier components and the non-linear contact forces and orbits at the rolling-bearing element are investigated.

Figures 13 and 14 show the non-linear amplitudes and the Fourier components relating to the unbalance and the radial clearance, respectively. Figures 15 illustrate the associated non-linear contact forces at the rolling-bearing element.

Firstly, the unbalance mass is increased from $1 g$ to $4 g$. In the simulations, a reduction of the unbalance mass appears to be an effective way to reduce the vibration level of the non-linear response and the subharmonic components if the rotation speed is higher than 1000rpm, as illustrated in Figures 13. 


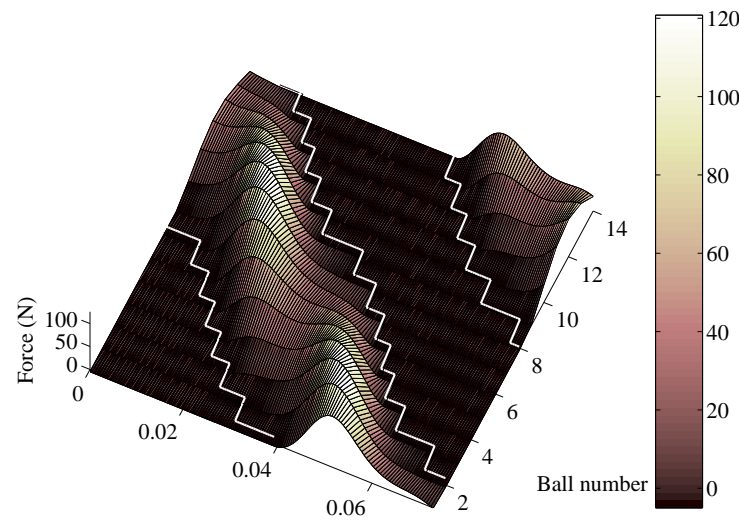

Time (s)

(a) $\omega=1895 \mathrm{rpm}$

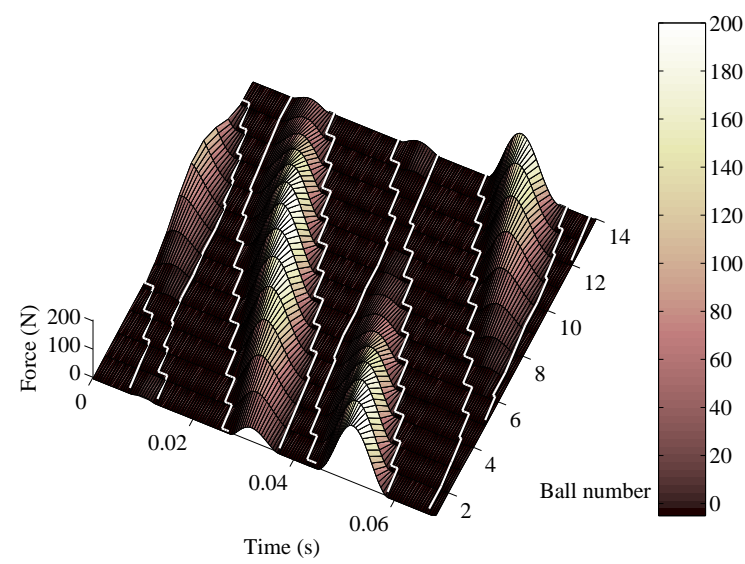

(c) $\omega=2030 \mathrm{rpm}$

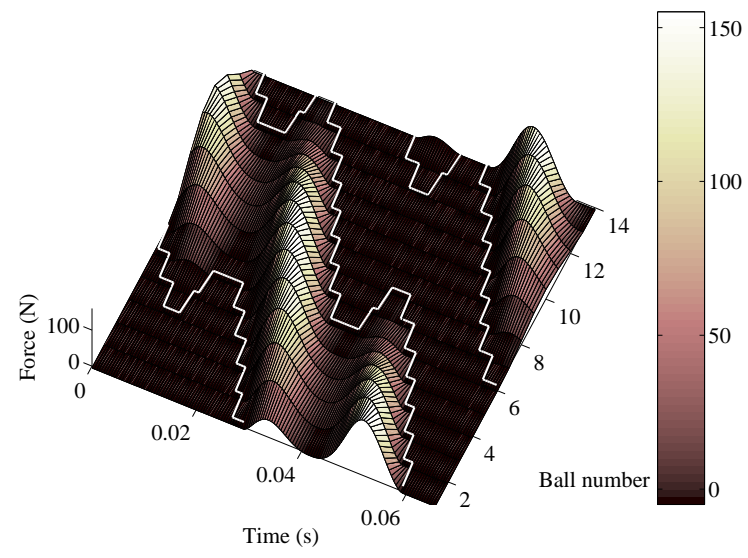

(e) $\omega=2115 \mathrm{rpm}$

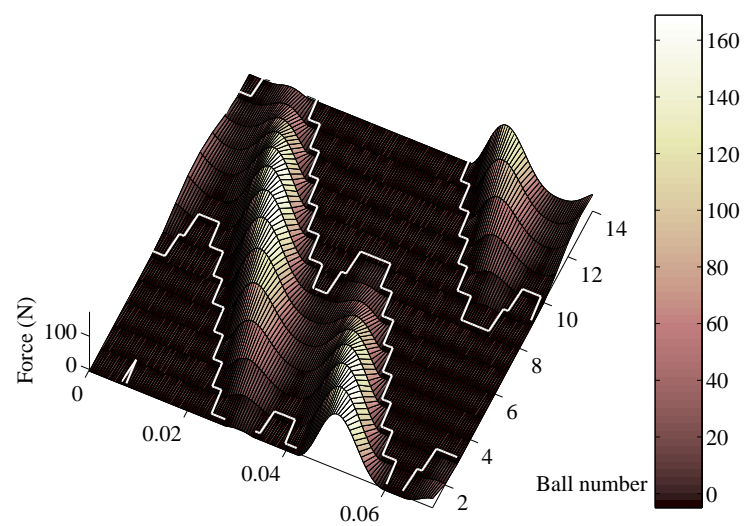

Time (s)

(b) $\omega=1990 \mathrm{rpm}$

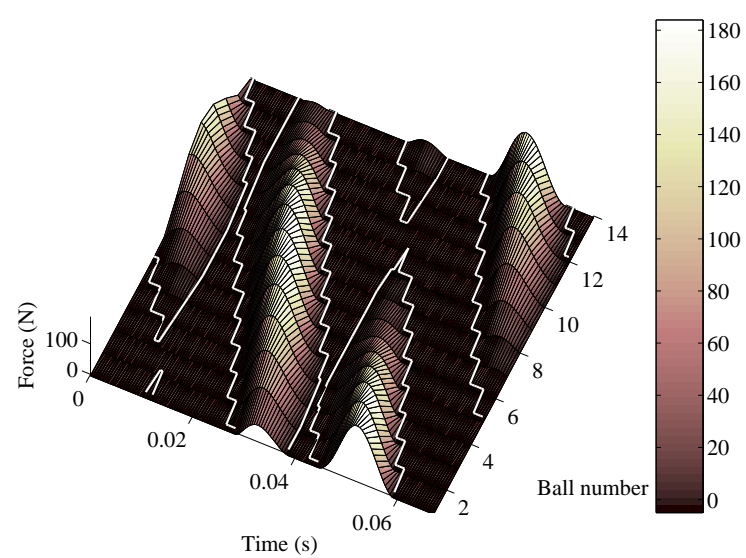

(d) $\omega=2066 \mathrm{rpm}$

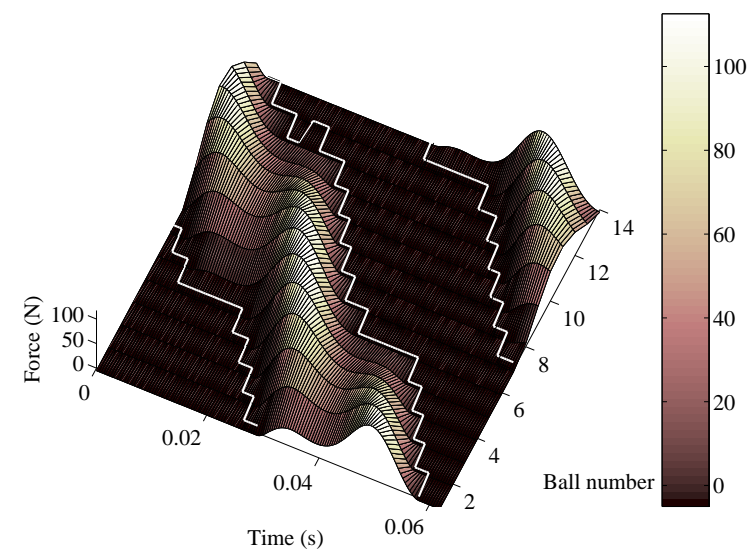

(f) $\omega=2244 \mathrm{rpm}$

Figure 8: Evolution of the contact on the balls for $\delta=10 \mu m$ and $m_{u}=2 g$ 


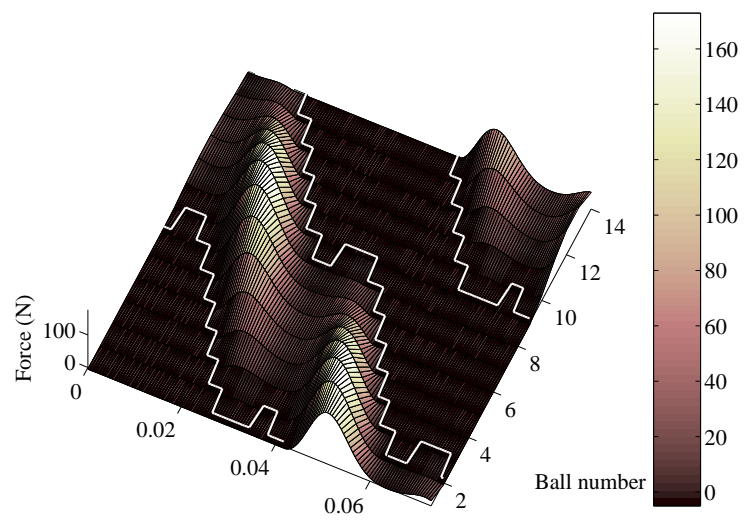

Time (s)

(a) $\omega=1895 \mathrm{rpm}$

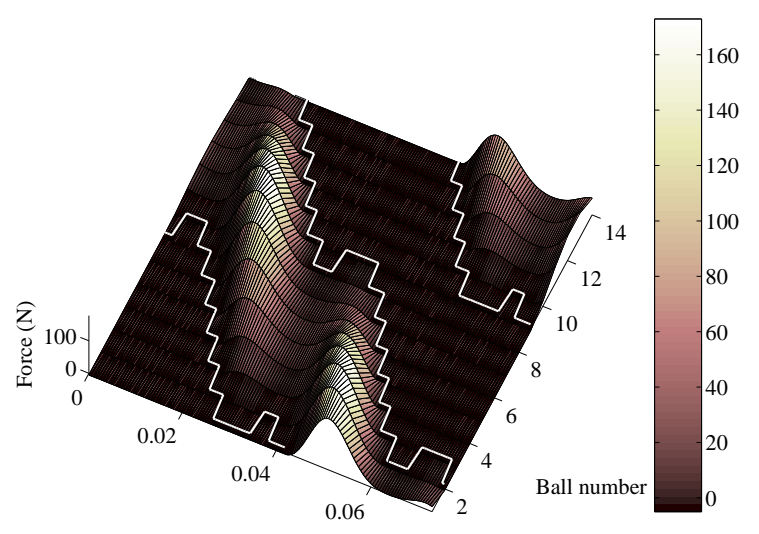

Time (s)

(c) $\omega=1947 \mathrm{rpm}$

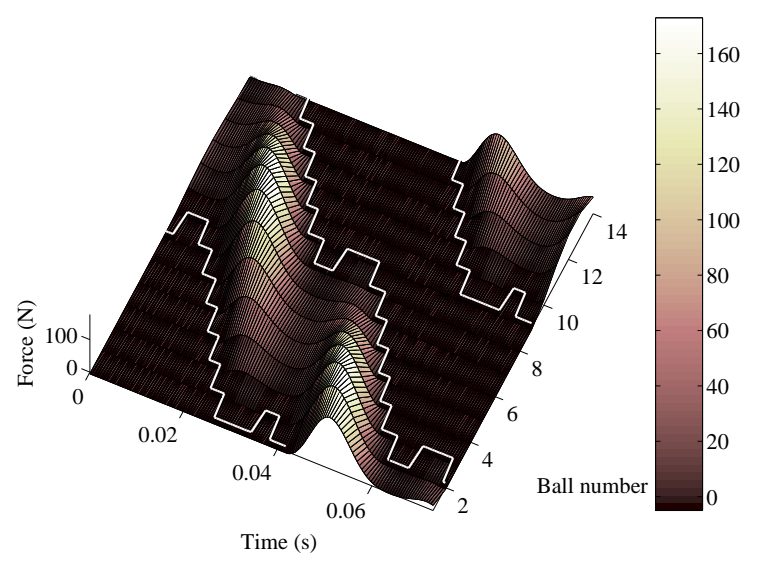

(e) $\omega=2253 \mathrm{rpm}$

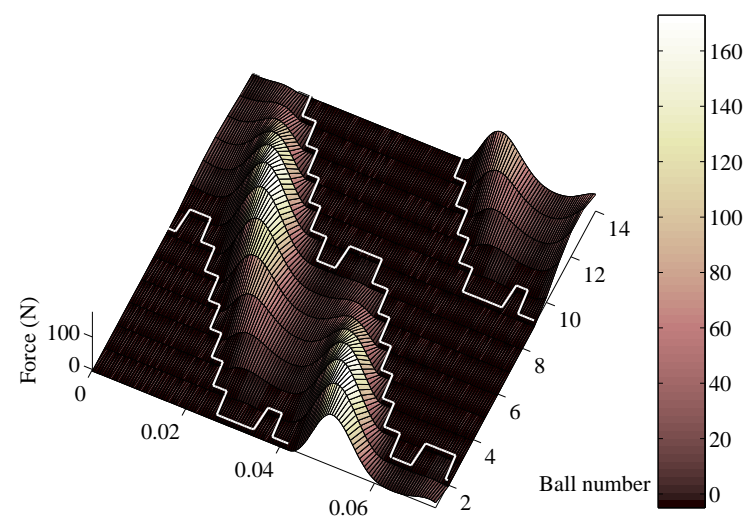

Time (s)

(b) $\omega=1919 \mathrm{rpm}$

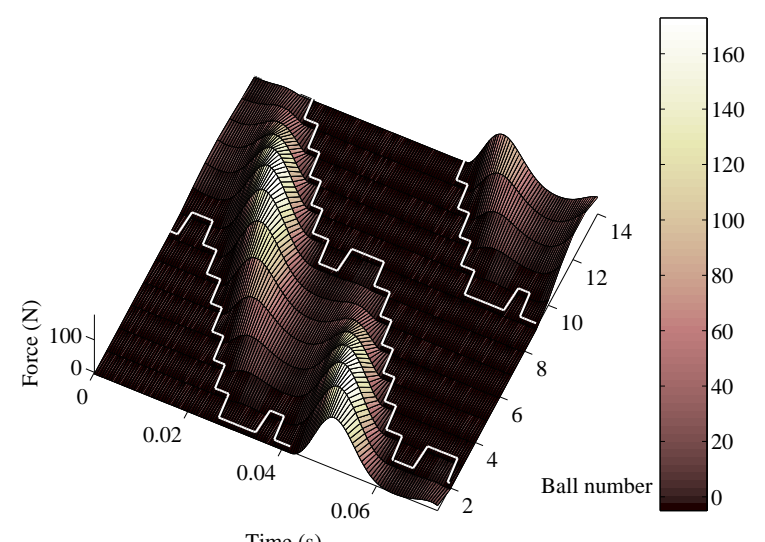

(d) $\omega=2204 r p m$

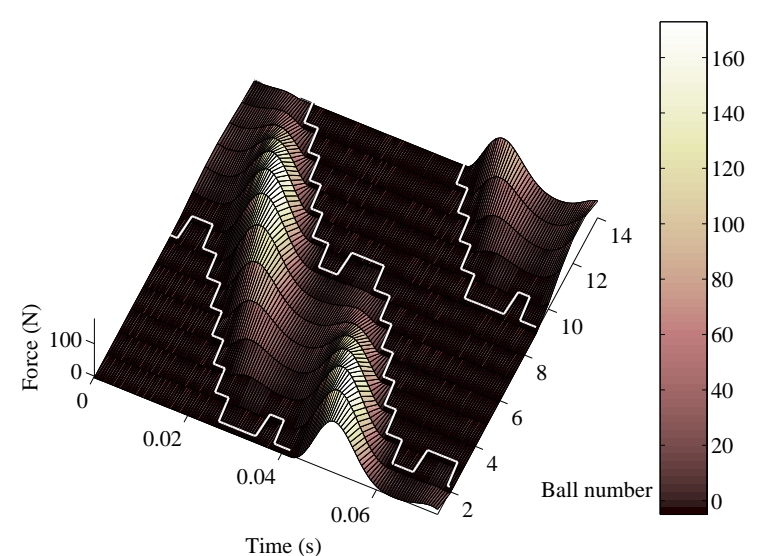

(f) $\omega=2403 \mathrm{rpm}$

Figure 9: Evolution of the contact on the balls for $\delta=10 \mu m$ and $m_{u}=4 g$ 


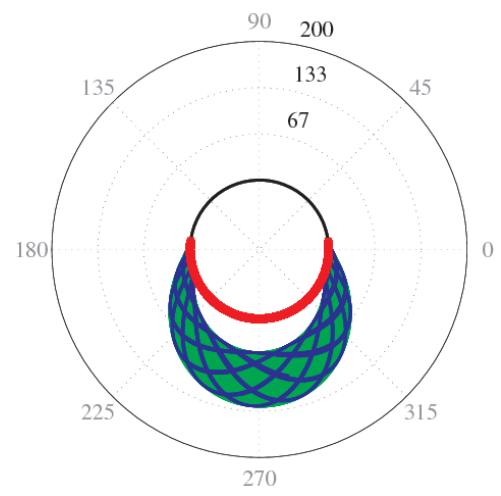

(a) $\omega=1895 \mathrm{rpm}$

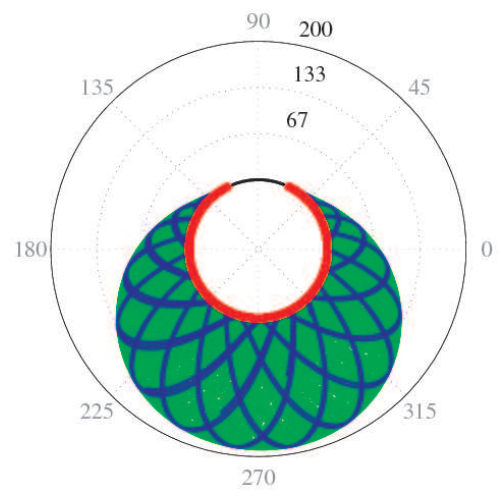

(d) $\omega=2066 \mathrm{rpm}$

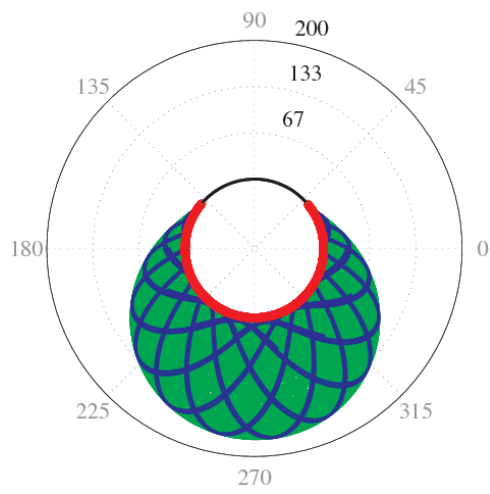

(b) $\omega=1990 \mathrm{rpm}$

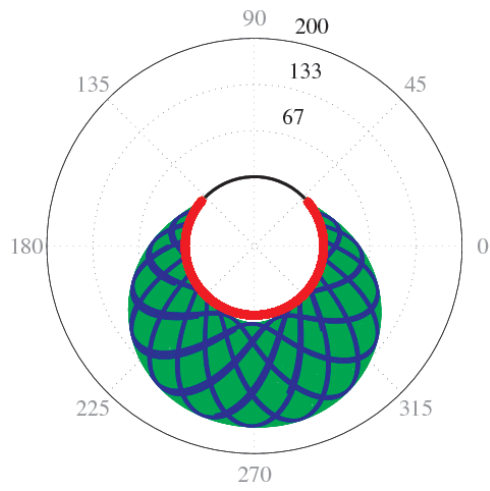

(e) $\omega=2115 \mathrm{rpm}$

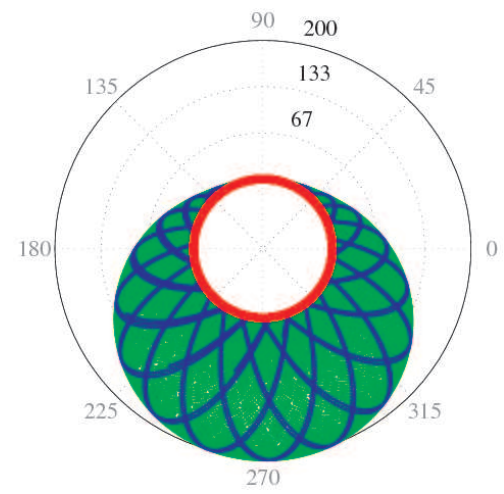

(c) $\omega=2030 \mathrm{rpm}$

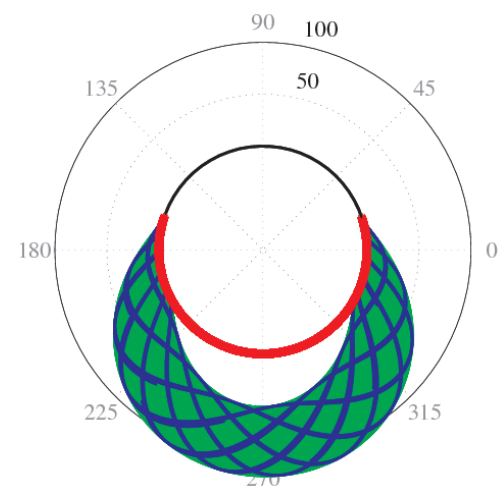

(f) $\omega=2244 r p m$

Figure 10: Evolution of the contact at the rolling-bearing for $\delta=10 \mu m$ and $m_{u}=2 g$ 


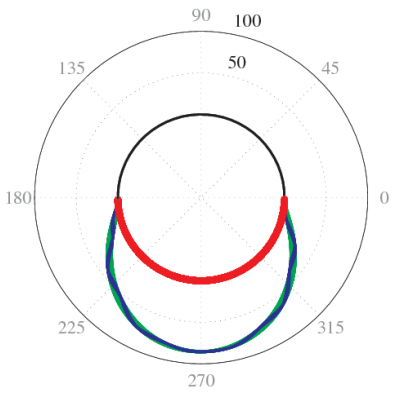

(a) $\omega=296 \mathrm{rpm}$

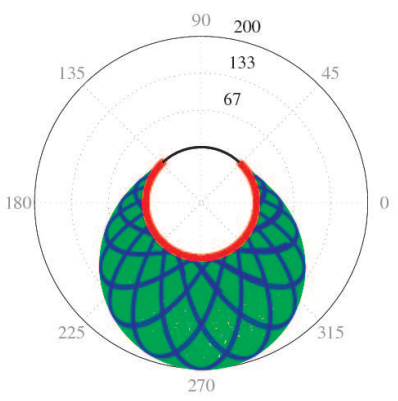

(e) $\omega=1916 \mathrm{rpm}$

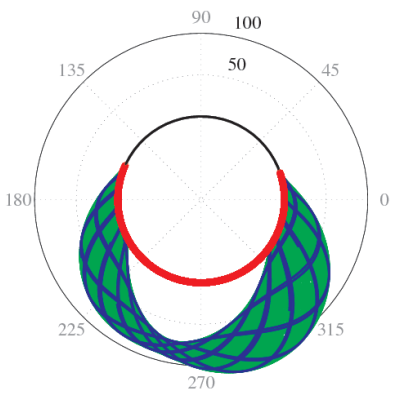

(i) $\omega=2703 \mathrm{rpm}$

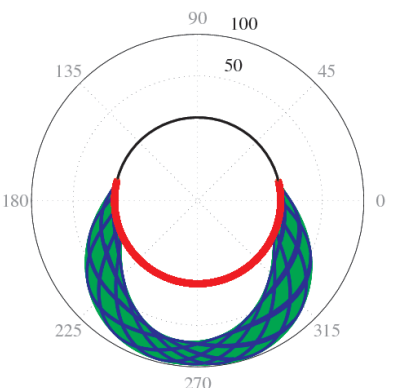

(b) $\omega=1595 \mathrm{rpm}$

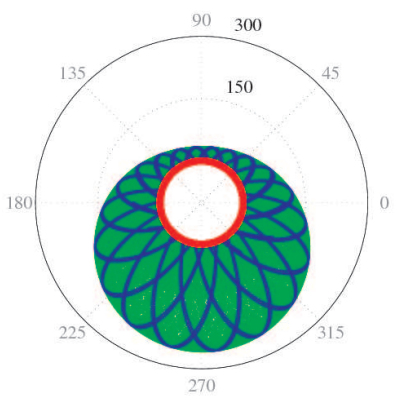

(f) $\omega=1996 \mathrm{rpm}$

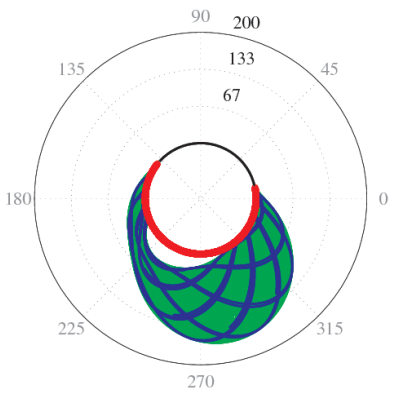

(j) $\omega=2890 \mathrm{rpm}$

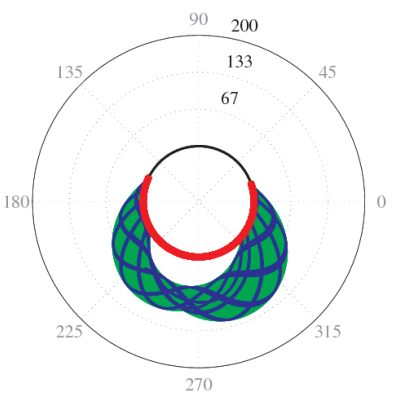

(c) $\omega=1696 \mathrm{rpm}$

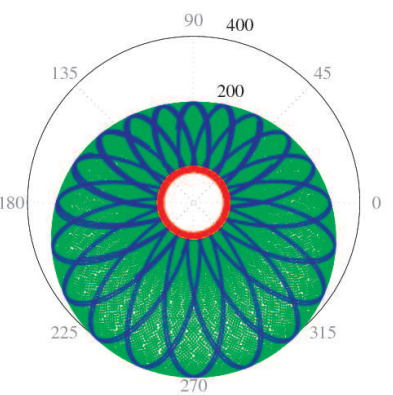

(g) $\omega=2195 \mathrm{rpm}$

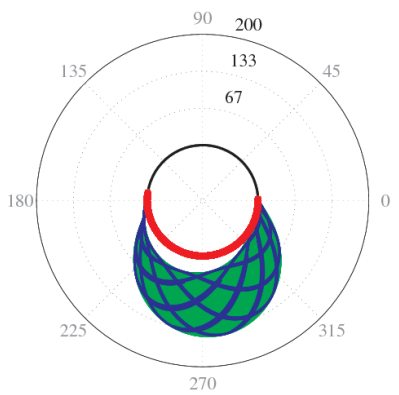

(k) $\omega=3004 r p m$

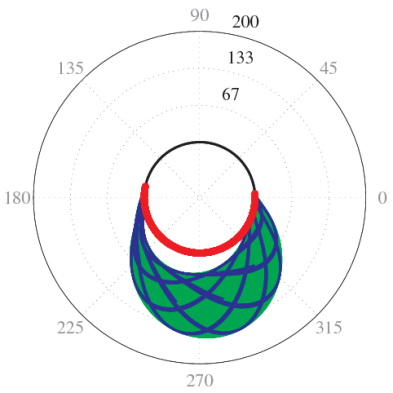

(d) $\omega=1797 \mathrm{rpm}$

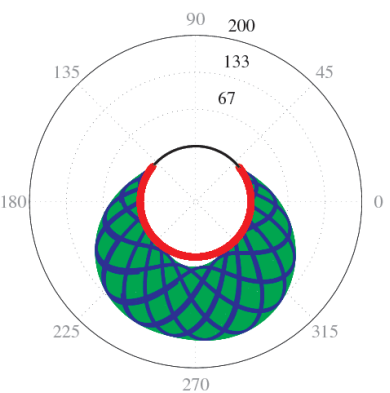

(h) $\omega=2253 \mathrm{rpm}$

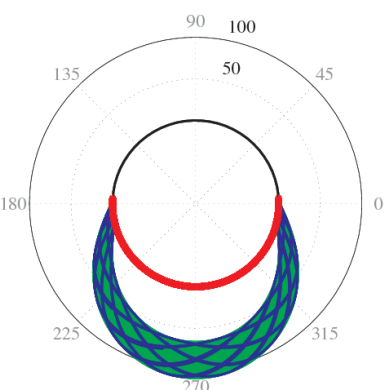

(l) $\omega=4000 \mathrm{rpm}$

Figure 11: Evolution of the contact at the rolling-bearing for $\delta=10 \mu \mathrm{m}$ and $m_{u}=4 \mathrm{~g}$ 


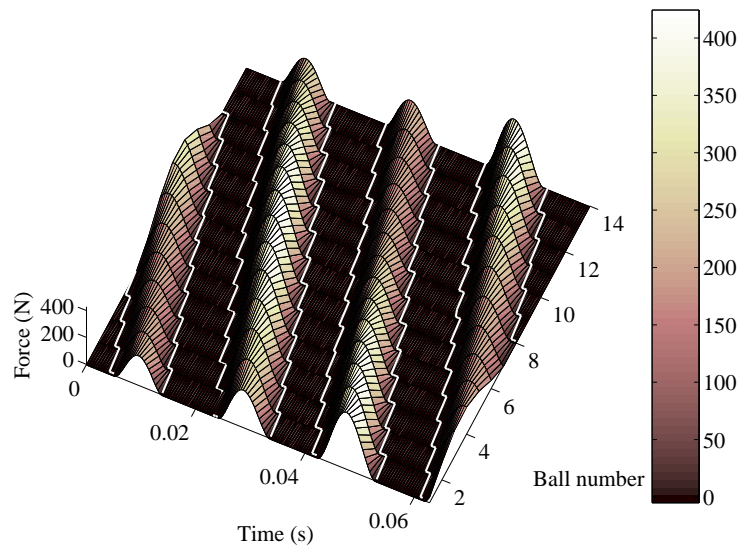

(a)

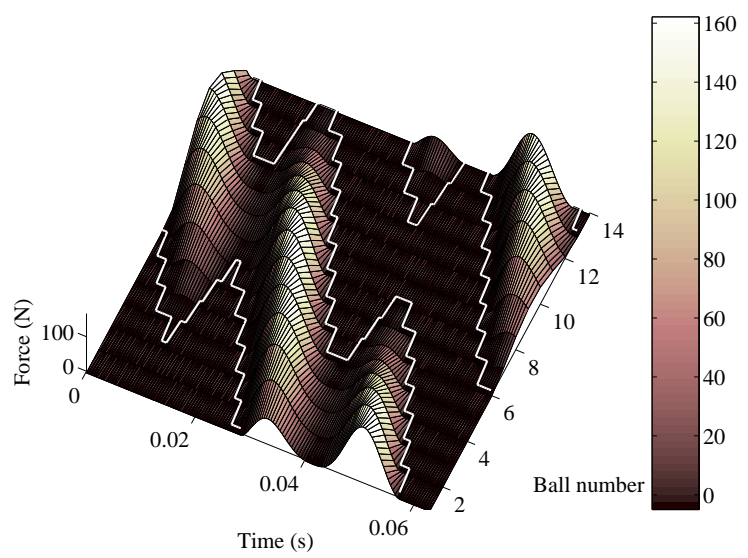

(c)

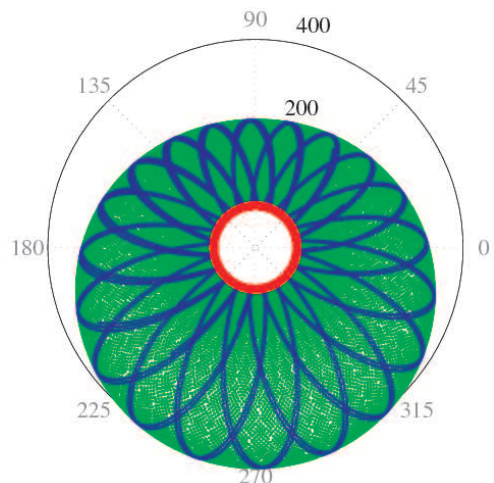

(b)

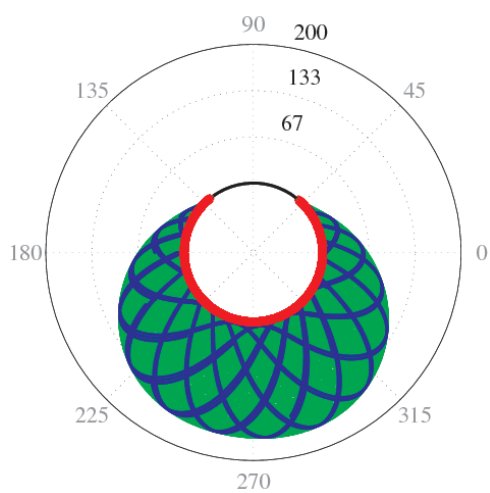

(d)

Figure 12: Contact comparison between a run-up and run-down for $m_{u}=4 g$ and $\delta=10 \mu m(\mathrm{a}-\mathrm{b})$ run-up (c-d) run-down 
Initially a softening-type nonlinearity, due to the non-linear contribution of the rolling bearing, is observed for low excitation levels (i.e. $m_{u}=[1 ; 2] \mathrm{g}$ ). This "softening effect"" is observed on the first and second orders around the first forward critical speed. For an excitation level of about 0.5 times the initial case (from $m_{u}=2 g$ to $m_{u}=1 g$ ), the peak of the response appears for a frequency lower than about $2 \%$ in comparison to the initial case (i.e $m_{u}=2 g$ ), even if the amplitude increases. For the two highest excitations (i.e. $m_{u}=3 g$ and $m_{u}=4 g$ ), a hardening-type nonlinearity is obtained with jumps. Jumps can be observed not only for the first order and the complete non-linear response (i.e. Composite Power), but also for the second harmonic component, as shown in Figure 13. For these two highest excitations, increases of about $2 \%$ and $8 \%$ respectively of the frequency of the maximum amplitude are observed. Moreover, an increase of the rotation speed interval where two stable solutions coexist is obtained due to the hardening-type nonlinearity of the rolling bearing. For low rotational speeds (i.e. $\omega=[0-1000] \mathrm{rpm}$ ), it is noted that the non-linear responses and the most important sub-harmonic component in the amplitudes (i.e. order 6) are not changed.

Secondly, Figures 14 give the non-linear amplitudes and the Fourier components for the radial clearance. In this example, the radial clearance is decreased from $30 \mu \mathrm{m}$ to $0 \mu \mathrm{m}$. It can be seen that that the radial clearance influences the critical speeds of the rotor. By increasing the radial clearance, a small softening-type nonlinearity can be observed. For the largest radial clearance $(30 \mu m)$, the peak of the response appears for a frequency lower by about $4 \%$ in comparison to the initial case (10 $\mu m$ ). However, the non-linear response and the subharmonic components appear to be very similar for the different radial clearances under study. Finally, Figures 15 illustrate the non-linear contact forces for the different unbalances and radial clearances for the rotation speed around 2000rpm. It should be noted that the unbalance mass influences the non-linear restoring forces at the rollingbearing element: increasing the mass unbalance allows the rotor to whirl in the cage with an increase of the non-linear contact forces. A reduction or increase of radial clearance has very little effect on the non-linear contact forces.

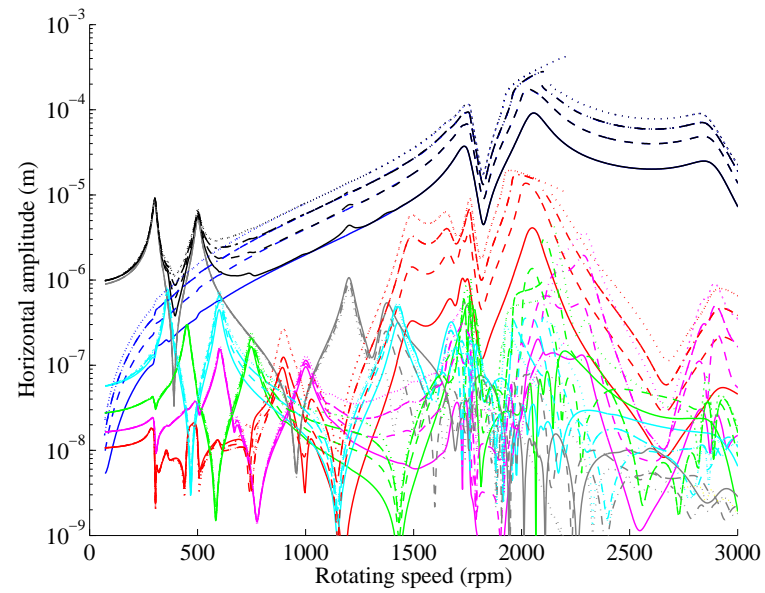

(a) at the non-linear rolling-bearing element

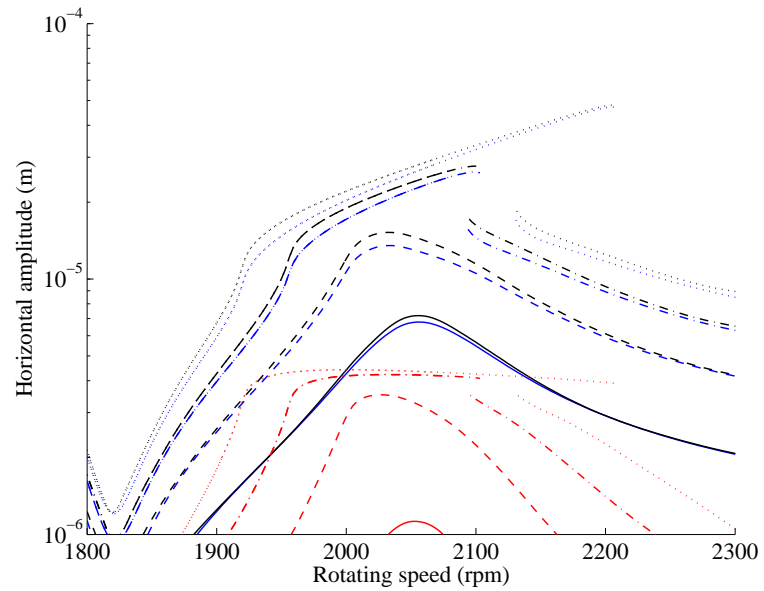

(b) zoom

Figure 13: Influence of the unbalance mass on the rotor unbalance responses (black=composite power, blue $=\operatorname{order} 1$, red $=$ order 2 , magenta $=$ order 3 , green $=\operatorname{order} 4$, cyan=order 5 , grey $=\operatorname{order} 6,-m_{u}=1 \mathrm{~g}$, $\left.--m_{u}=2 g,-. m_{u}=3 g, \cdots m_{u}=4 g\right)$ 


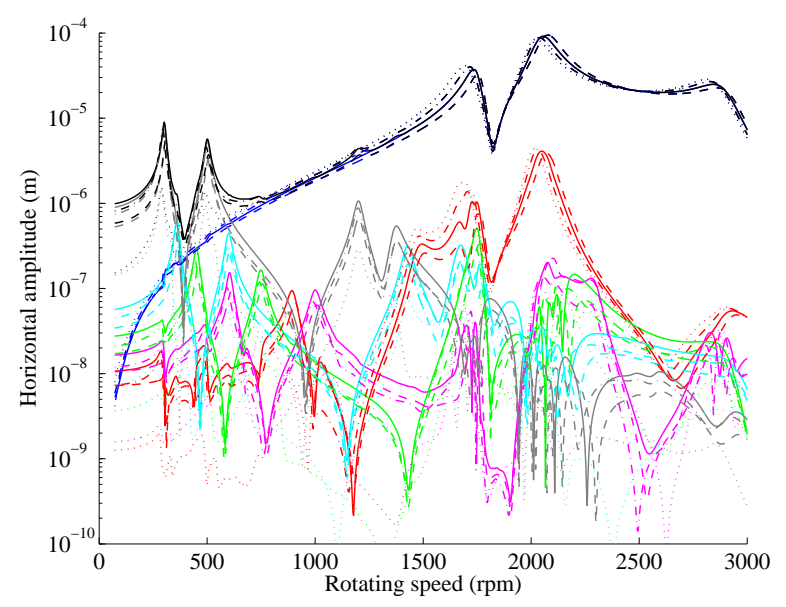

(a) at the non-linear rolling-bearing element

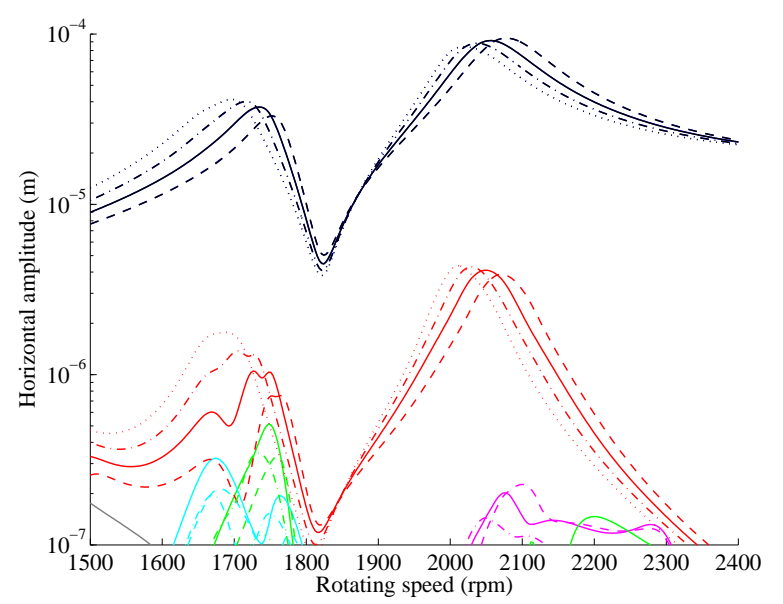

(b) zoom

Figure 14: Influence of the clearance on the rotor unbalance responses (black=composite power, blue $=$ order 1 , red $=$ order 2 , magenta $=\operatorname{order} 3$, green $=\operatorname{order} 4$, cyan=order 5 , grey=order $6,-\delta=$ $10 \mu m,--\delta=0 \mu m,-. \delta=20 \mu m, \cdots \delta=30 \mu m)$

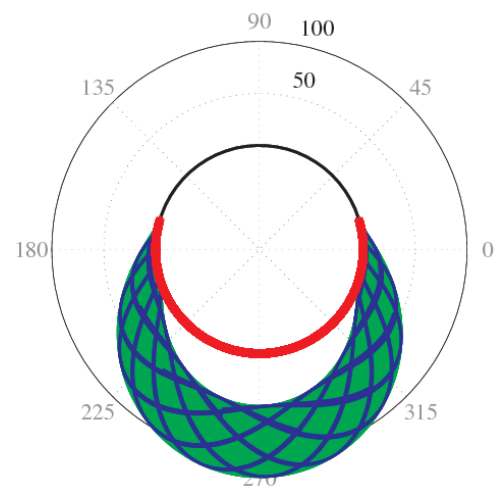

$\delta=0 \mu m$ and $m_{u}=1 g$

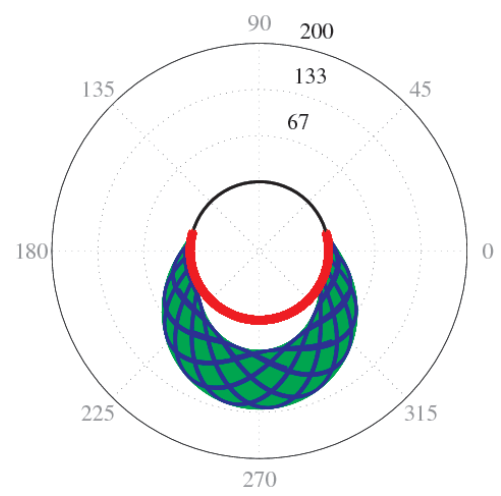

$\delta=10 \mu m$ and $m_{u}=1 g$

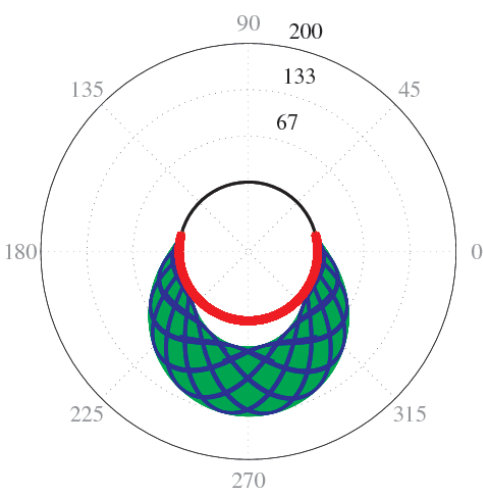

$\delta=20 \mu m$ and $m_{u}=1 g$

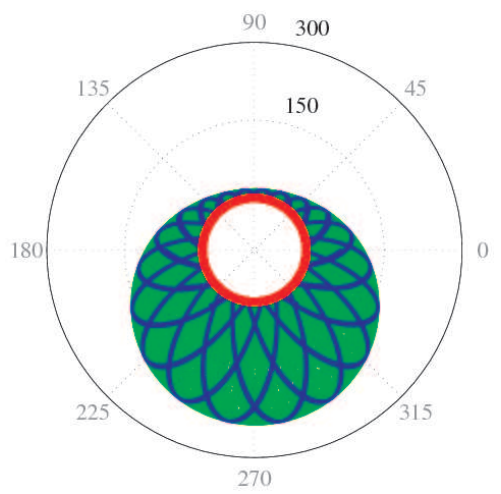

$\delta=10 \mu m$ and $m_{u}=3 g$

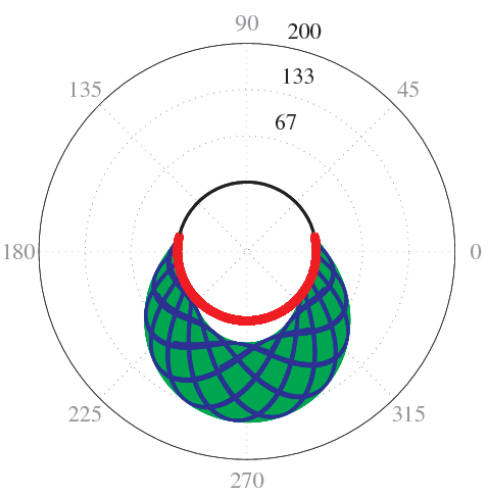

$\delta=30 \mu m$ and $m_{u}=1 g$

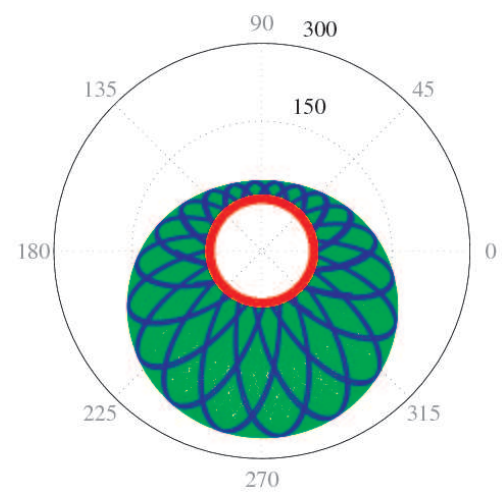

$\delta=10 \mu m$ and $m_{u}=4 g$

Figure 15: Evolution of the contact at the rolling-bearing 


\section{Conclusion}

This Harmonic Balance Method with a condensation procedure was developed in order to analyze the non-linear behavior of a rotor system with a rolling bearing elements. In this study, twelve harmonic components were chosen in order to approximate the non-linear response. The non-linear elements are both due to the radial clearance and the Herztian contact between races and rolling elements.

The results demonstrated that the non-linear unbalance response and the associated orbits can be very complex due to the non-linear elements and the relative contribution of unbalance forces and radial clearance: the $n^{\text {th }}$ Fourier components can make a significant contribution when the rotor passes through the $\frac{1}{n}$ sub-critical resonances and critical speeds. In particular, the $2 \times$ and $3 \times$ and $6 \times$ superharmonic frequency components and $\frac{1}{2}, \frac{1}{3}$ and $\frac{1}{6}$ sub-critical resonances are significant. It appears that the vibration amplitudes in the sub-critical and critical resonances depend on the radial clearance and unbalance mass. If the unbalance mass and radial clearance remain constant during operation of rotor, the $1 \times, 2 \times$ and $3 \times$ amplitude may change during run-up or rundown due to the occurrence of the jump phenomenon.

The changes in the non-linear contacts at each rolling bearing element were examined in details, with particular attention given to when the rotor exceeds critical speeds. It was shown that the contact evolution for each ball-bearing can be very simple even if the non-linear behavior of the rotor and the associated orbits are complex. Finally, it was shown that mass unbalance and radial clearance affect the non-linear contact forces and the whirling motion of the rotor at the rolling-bearing element. In particular, it was shown that increasing small excitation levels leads to an initial softening-type nonlinearity, which turns into hardening-type for higher excitations.

\section{Nomenclature}

$\mathrm{X}$ displacement vector

$\dot{\mathrm{X}} \quad$ velocity vector

$\ddot{\mathrm{X}} \quad$ acceleration vector

M mass matrix of the rolling-bearing rotor system

K stiffness matrix of the rolling-bearing rotor system

G gyroscopic matrix of the rolling-bearing rotor system

C damping matrix of the rolling-bearing rotor system

$\mathbf{F}_{L} \quad$ vector of the weight and unbalance forces

$\mathbf{F}_{N L} \quad$ vector of the non-linear forces due to the rolling-element bearing

$m \quad$ order of the Fourier series

$\mathbf{A}_{k} \quad$ Fourier coefficients of the sinus function for the $k^{t h}$ order

$\mathbf{B}_{k} \quad$ Fourier coefficients of the cosinus function for the $k^{\text {th }}$ order

$\mathbf{Y}^{p} \quad p$ linear degrees of freedom of the rolling bearing rotor system

$\mathbf{Y}^{q} \quad q$ non-linear degrees of freedom of the rolling bearing rotor system

$\mathbf{B}_{k}^{q} \quad$ Fourier coefficients of the sinus function for the $k^{t h}$ order of the non-linear dof

$\mathbf{A}_{k}^{q} \quad$ Fourier coefficients of the cosinus function for the $k^{t h}$ order of the non-linear dof

$\mathbf{B}_{k}^{p} \quad$ Fourier coefficients of the sinus function for the $k^{t h}$ order of the linear dof

$\mathbf{A}_{k}^{p} \quad$ Fourier coefficients of the cosinus function for the $k^{\text {th }}$ order of the linear dof 


\section{References}

[1] Chinta, M., and Palazzolo, A. B., 1998. "Stability and bifurcation of rotor motion in a magnetic bearing”. Journal of Sound and Vibration, 214, p. 793-803.

[2] Virgin, L. N., Walsh, T. F., and Knight, J. D., 1995. "Nonlinear behavior of a magnetic bearing system". Journal of Sound and Vibration, 117, p. 582-588.

[3] Ji, J. C., and Leung, A. Y. T., 2003. "Non-linear oscillations of a rotor-magnetic bearing system under superharmonic resonance conditions". International Journal of Non-Linear Mechanics, 38, p. $829-835$.

[4] Erich, F. E., 1992. Handbook of Rotordynamics. McGraw-Hill.

[5] Childs, D., 1993. Turbomachinery Rotordynamics: Phenomena, Modeling, and Analysis. WileyInterscience.

[6] Yamamoto, T., and Ishida, Y., 2001. Linear and Nonlinear Rotordynamics: a Modern Treatment with Applications. Wiley and Sons.

[7] Ding, Q., and Leung, A. Y. T., 2003. "Non-stationary processes of rotor/bearing system in bifurcations". Journal of Sound and Vibration, 268, p. 33-48.

[8] Hahn, E. J., and Chen, P. Y., 1994. "Harmonic balance analysis of general squeeze film damped multidegree-of-freedom rotor bearing systems". Journal of Tribology, 116, pp. 499-507.

[9] Harris, T. A., 2001. Rolling Bearing Analysis, 4 ed. John Wiley and Sons.

[10] Ehrich, F. F., and O’Connor J. J., 1967. "stator whirl rotors in bearing clearance”. ASME Journal of Engineering for Industry, pp. 381-390.

[11] Ehrich, F. F., 1988. "High order subharmonic response if high speed rotors in bearing clearance". ASME Journal of Vibration, Acoustics, Stress, and Reliability in Design, 110, pp. 9-16.

[12] Sunnersjo, C. S., 1978. "Ball passage vibrations of rolling bearings". Journal of Sound and Vibration, 58, pp. 363-373.

[13] Choi, Y. S., and Noah, S. T., 1987. "Nonlinear steady state response of a rotor-support system". ASME Journal of Vibration, Accoustics, Stress and Reliability in Design, 109(255-261).

[14] Tiwari, M., Gupta, K., and Prakash, O., 2000. "Effect of a ball bearing on the dynamics of a balanced horizontal rotor". Journal of Sound and Vibration, 238(5), pp. 723-756.

[15] Tiwari, M., and Gupta, K., 2000. "Dynamics response of an unbalanced rotor supported on ball bearings". Journal of Sound and Vibration, 238(5), pp. 757-779.

[16] Harsha, S. P., Sandeep, K., and Prakash, R., 2004. "Non-linear dynamic behaviors of rolling element bearings due to surface waviness". Journal of Sound and Vibration, 272, pp. 557-580. 
[17] Harsha, S. P., 2006. "Nonlinear dynamic response of a balanced rotor supported by rolling element bearings due to radial internal clearance effect ". Mechanism and Machine Theory, 41(6), pp. 688-706.

[18] Mevel, B., Guyader, J. L., 2008. "Experiments on routes to chaos in ball bearings". Journal of Sound and Vibration, 318(549-564).

[19] Nataraj, C., and Harsha.,S. P., 2008. "The effect of bearing cage run-out on the nonlinear dynamics of a rotation shaft". Communications in Nonlinear Science and Numerical Simulation , 13, pp. $822-838$.

[20] Jang, G., and Jeong, S. W., 2004. "Vibration analysis of a rotation system due to the effect of ball bearing waviness". Journal of Sound and Vibration, 269, pp. 709-726.

[21] Nayfeh, A. H., and Mook, D. T., 1995. Nonlinear oscillations. John Wiley \& Sons.

[22] Sinou, J.-J., and Lees, A., 2007. "A non-linear study of a cracked rotor". Journal of European Mechanics - A/Solids, 26(1), pp. 152-170.

[23] Cameron, T. M., and Griffin, J. H., 1989. "An alternating frequency time domain method for calculating the steady state response of nonlinear dynamic systems". ASME Journal of Applied Mechanics, 56, pp. 149-154.

[24] Broyden, C. G., 1965. "A class of methods for solving nonlinear simultaneous equations". Mathematics of Computations, 19, pp. 577-593.

[25] Sinou, J.-J., 2008. "Detection of cracks in rotor based on the $2 X$ and $3 X$ super-harmonic frequency components and the crack-unbalance interactions". Communications in Nonlinear Science and Numerical Simulation, 13, pp. 2024-2040. 